\title{
Systematic analysis of JmjC gene family and stress-response expression of KDM5 subfamily genes in Brassica napus
}

\author{
Xinghui He ${ }^{1,2,3}$, Qianwen Wang ${ }^{3}$, Jiao Pan ${ }^{1,2,3}$, Boyu Liu ${ }^{1,2,3}$, Ying Ruan ${ }^{1,2,3}$, Yong Huang ${ }^{\text {Corresp. 1, 2, } 3}$ \\ ${ }^{1}$ Key Laboratory of Crop Epigenetic Regulation and Development, Hunan Province, Changsha, China \\ ${ }^{2}$ Key Laboratory of Plant Genetics and Molecular Biology of Education Department, Changsha, Hunan Province, China \\ 3 College of Bioscience and Biotechnology, Hunan Agricultural University, Changsha, Hunan Province, China \\ Corresponding Author: Yong Huang \\ Email address: yonghuang@hunau.edu.cn
}

Background. Jumonji C ( JmjC ) proteins exert critical roles in plant development and stress response through the removal of lysine methylation from histones. Brassica napus, which originated from spontaneous hybridization by Brassica rapa and Brassica oleracea, is the most important oilseed crop after soybean. In JmjC proteins of Brassica species, the structure and function and its relationship with the parents and model plant Arabidopsis thaliana remain uncharacterized. Systematic identification and analysis for JmjC family in Brassica crops can facilitate the future functional characterization and oilseed crops improvement.

Methods. Basing on the conserved JmjC domain, JmjC homologs from the three Brassica species, $B$. rapa $(A A), B$. oleracea (CC) and $B$. napus, were identified from the Brassica database. Some methods, such as phylogenic analysis, chromosomal mapping, HMMER searching, gene structure display and Logos analysis, were used to characterize relationships of the JmjC homologs. synonymous and nonsynonymous nucleotide substitutions were used to infer the information of gene duplication among homologs. Then, the expression level of BnKDM5 subfamily genes were checked under abiotic stress by qRT-PCR.

Results. $65 \mathrm{Jmj} C$ genes were identified from $B$. napus genome, 29 from $B$. rapa, and 23 from $B$. oleracea. These genes were grouped into seven clades base on the phylogenetic analysis, and their catalytic activities of demethylation were predicted. The average retention rate of $B$. napus Jmj $C$ genes ( $B$. napus JmjC gene from B. rapa (93.1\%) and B. oleracea (82.6\%)) exceeded whole genome level. JmjC sequences demonstrated high conservation in domain origination, chromosomal location, intron/exon number and catalytic sites. The gene duplication events were confirmed among the homologs. Furthermore, BrKDM5 subfamily genes were examined under stress conditions. A lot of BrKDM5 subfamily genes show higher expression under drought and $\mathrm{NaCl}$ treatment, but only a few genes are involved in high temperature stress.

Conclusions. This study provides the first genome-wide characterization of JmjC genes in Brassica species. The $B n J m j C$ exhibits higher conservation during the formation process of allotetraploid than the average retention rates of whole $B$. napus genome. Furthermore, expression profiles indicated that BnKDM5 subfamily genes are involved in stress response to salt, drought and high temperature. 
1

2

3

4

5

61 College of Bioscience and Biotechnology, Hunan Agricultural University, Changsha, China

$7 \quad 2$ Key Laboratory of Crop Epigenetic Regulation and Development in Hunan Province, Changsha, China

83 Key Laboratory of Plant Genetics and Molecular Biology of Education Department in Hunan Province, Changsha, China

9

10

11

12 Email addresses for the authors:

13 Xinghui He: hxhlovely@outlook.com

14 Qianwen Wang: sandyqianwenwang@,163.com

15 Jiao Pan: jiaozijiaoa@163.com

16 Boyu Liu: liuboyu@hunau.edu.cn

17 Ying Ruan: yingruan@hotmail.com

18 Yong Huang: yonghuang@hunau.edu.cn

19
Systematic analysis of $\mathrm{JmjC}$ gene family and

genes in Brassica napus 
20 Abstract

21 Background. Jumonji C $(\mathrm{JmjC})$ proteins exert critical roles in plant development and stress response through

22 the removal of lysine methylation from histones. Brassica napus, which originated from spontaneous

23 hybridization by Brassica rapa and Brassica oleracea, is the most important oilseed crop after soybean. In

$24 \mathrm{JmjC}$ proteins of Brassica species, the structure and function and its relationship with the parents and model

25 plant Arabidopsis thaliana remain uncharacterized. Systematic identification and analysis for JmjC family in

26 Brassica crops can facilitate the future functional characterization and oilseed crops improvement.

27 Methods. Basing on the conserved JmjC domain, JmjC homologs from the three Brassica 28 species, B. rapa (AA), B. oleracea (CC) and B. napus, were identified from the Brassica database. Some 29 methods, such as phylogenic analysis, chromosomal mapping, HMMER searching, gene structure display and 30 Logos analysis, were used to characterize relationships of the JmjC homologs. synonymous and 31 nonsynonymous nucleotide substitutions were used to infer the information of gene duplication among 32 homologs. Then, the expression level of BnKDM5 subfamily genes were checked under abiotic stress by qRT33 PCR.

34 Results. $65 \mathrm{JmjC}$ genes were identified from B. napus genome, 29 from B. rapa, and 23 from B. oleracea.

35 These genes were grouped into seven clades base on the phylogenetic analysis, and their catalytic activities of 36 demethylation were predicted. The average retention rate of B. napus JmjC genes (B. napus JmjC gene from B. 37 rapa (93.1\%) and B. oleracea (82.6\%)) exceeded whole genome level. JmjC sequences demonstrated high 38 conservation in domain origination, chromosomal location, intron/exon number and catalytic sites. The gene 39 duplication events were confirmed among the homologs. Furthermore, BrKDM5 subfamily genes were 40 examined under stress conditions. A lot of BrKDM5 subfamily genes show higher expression under drought 41 and $\mathrm{NaCl}$ treatment, but only a few genes are involved in high temperature stress.

42 Conclusions. This study provides the first genome-wide characterization of JmjC genes in Brassica species.

43 The BnJmjC exhibits higher conservation during the formation process of allotetraploid than the average

44 retention rates of whole B. napus genome. Furthermore, expression profiles indicated that BnKDM5 45 subfamily genes are involved in stress response to salt, drought and high temperature. 


\section{INTRODUCTION}

47 Epigenetics refers to heritable change for gene function that occurs without a change in DNA

48 sequence and can dynamically regulate global gene expression through reversible chemical

49 modifications on DNA and histones in eukaryotic chromatin (He \& Cole, 2015). Epigenetic

50 regulation mainly includes acetylation, phosphorylation, histone methylation, DNA methylation,

51 and small non-coding RNAs. Histone modification is an important epigenetics mechanism.

52 Various post-translational covalent modifications, which primarily occur on histone (H3, H4,

$53 \mathrm{H} 2 \mathrm{~A}$, and H2B) lysines and arginines residues, form "histone code" to regulate various biological

54 processes (Bannister \& Kouzarides, 2011). Histone methylation is usually catalyzed by three

55 protein families of histone methyltransferases: protein arginine methyltransferase family, $\mathrm{Su}$

56 (var)3-9, Enhancer-of-zeste and Trithorax (SET) domain family, and telomeric silencing

57 disruptor that is also known as DOT1-Like (Kmt4/DOT1L) (Greer \& Shi, 2012). Histone lysine

58 methylation, playing many different roles in biological processes ranging from heterochromatin

59 formation to transcription regulation, is dynamically regulated by histone lysine

60 methyltransferases (KMTs) and histone lysine demethylases (KDMs), and can be distinguished

61 depending on the position of lysine residue and the number of added methyl groups in lysine

62 residues, which carry mono-, di-, or tri-methylated groups (Liu et al., 2010).

63 Histone modifications can influence gene expression to regulate the plant response to stress,

64 including cold, freezing, saline, drought and submergence (Bej \& Basak, 2017). Genome-wide

$65 \mathrm{H} 3 \mathrm{~K} 4$ methylation patterns (H3K4me1, $\mathrm{H} 3 \mathrm{~K} 4 \mathrm{me} 2$ and $\mathrm{H} 3 \mathrm{~K} 4 \mathrm{me} 3)$ show dynamic responses to

66 dehydration stress in Arabidopsis thaliana (van Dijk et al., 2010). Arabidopsis ATX1, H3K4me3,

67 is involved in dehydration response through ABA-dependent and -independent pathways (Ding et

68 al., 2011). Under drought stress, H3K4me3 enrichment is correlated with the activation of

69 Arabidopsis drought stress-responsive genes, such as RD29A and RD20 (Kim et al., 2012; Qiao \& 
70 Fan, 2011). H3K4me3 can be maintained at low levels after rehydration and which could

71 function as an epigenetic mark of drought stress memory (Kim et al., 2012). Under heat stress,

$72 \mathrm{H} 3 \mathrm{~K} 4$ methylation accumulates to activate gene expression and can be sustained after heat stress

73 to positively respond on a future stress incident (Lamke et al., 2016). Rice genome-wide

74 H3K4me3 profiling showed positively correlation with the transcript level of drought stress-

75 responsive genes (Zong et al., 2013). Histone methylation is also a reversible process regulated

76 by methyltransferases and three distinct classes of demethylases. KDMs mainly consist of LSD1/

77 KDM1s (Lysine specific demethylase 1) and JmjC-domain enzymes, which both utilize oxidative

78 mechanisms. JmjC, a highly conserved domain, was first reported by Takeuchi and colleagues in

791995 and was named as JmjC domain in 2000 (Balciunas \& Ronne, 2000; Takeuchi et al., 1995).

80 This domain carries eight $\beta$-sheets forming enzymatically-active pocket with three conserved and

81 necessary amino-acid residues for binding with Fe (II) cofactor and two additional residues for

82 binding with $\alpha$-ketoglutarate $(\alpha \mathrm{KG})$ (Chen et al., 2006; Klose et al., 2006). Arabidopsis JmjC

83 proteins are divided into five subfamilies: KDM4/JHDM3 (AtJMJ11-13), KDM5/JARID1

84 (AtJMJ14-19), JMJD6 (AtJMJ21/22), KDM3/JHDM2 (AtJMJ24-29) and JmjC domain-only

85 (AtJMJ20 and AtJMJ30-32) (Luo et al., 2013). The H3K4 methylases and demethylases

86 dynamically balance the H3K4 methylation status among H3K4me1, H3K4me2 and H3K4me3,

87 to maintain the optimum level of $\mathrm{H} 3 \mathrm{~K} 4$ methylation and adapt to external environment. KDM5 is

88 a specific subfamily that specifically removes H3K4 methylation modifications. However, most

89 reports on $\mathrm{H} 3 \mathrm{~K} 4$ demethylase functions were mainly focused on regulating plant development.

90 For example, AtJMJ14/PKDM7B, a histone H3K4 demethylase, represses floral integrators

91 Flowering Locus $T(F T), A P 1, S O C 1$ and $L F Y$ during vegetative growth (Lu et al., 2010; Yang et

92 al., 2010). AtJMJ15 regulates flowering time by demethylating H3K4me3 at Flowering Locus C

93 (FLC) chromatin (Yang et al., 2012b). AtJMJ18 is dominantly expressed in companion cells 
94 exhibiting $\mathrm{H} 3 \mathrm{~K} 4 \mathrm{me} 3$ and $\mathrm{H} 3 \mathrm{~K} 4 \mathrm{me} 2$ demethylase activity of $F L C$. atjmj18 mutation results in a

95 weak late-flowering phenotype, and its overexpression induces early-flowering (Yang et al.,

96 2012a). Moreover, the overexpression of AtJMJ15 may regulate gene expression that enhances

97 stress tolerance (Shen et al., 2014). Although several functions of H3K4 methylation

98 modifications in response to abiotic stresses have been reported, only a few were evaluated.

99 Brassica species might have diverged from a common ancestor with an Arabidopsis lineage from

100 14.5-20.4 million years ago (Yang et al., 1999). Allotetraploid species Brassica napus (oilseed

101 rape, $\mathrm{AACC}, 2 \mathrm{n}=38$ ) originated from interspecific spontaneous hybridization between Brassica

102 rapa $(\mathrm{AA}, 2 \mathrm{n}=20)$ and Brassica oleracea $(\mathrm{CC}, 2 \mathrm{n}=18)$ (Yang et al., 2010). The protein

103 organization and function of JmjC domain in Brassica species and its relative relationship with

104 model plant Arabidopsis remain uncharacterized. B. napus is currently the most important oilseed

105 crop, preceded only by soybean. However, B. napus is vulnerable to abiotic stress that limits its

106 growth and productivity and reduces its economic benefits. KDM5/JARID1 subfamily may

107 regulate many abiotic stress responses genes through down-regulated $\mathrm{H} 3 \mathrm{~K} 4 \mathrm{me} 3$ and $\mathrm{H} 3 \mathrm{~K} 4 \mathrm{me} 2$

108 but the roles of $\mathrm{H} 3 \mathrm{~K} 4$ demethylation in abiotic stress remain unknown.

109 MATERIALS AND METHODS

110 Identification of Jmjc Proteins and Chromosomal Map Construction

111 The JmjC protein sequences of Arabidopsis thaliana (AtJMJ11-22 and AtJMJ24-32) were

112 obtained as our previously described by Huang et al in 2016 (Supplement File 1), and these

113 sequences were used as queries to BLASTp JmjC proteins of B. rapa, B. oleracea and B. napus

114 in Brassica database (http://brassicadb.org/brad/blastPage.php). Oryza sativa sequences were

115 retrieved from Phytozome database (Version 12). The result sequences of BLASTp were

116 confirmed using both SMART (HMMER) and NCBI (BLASTp) with default parameters, and

117 proteins without JmjC domain were excluded. The loci information of JmjC gene was used to 
118 generate chromosome maps by the Mapchart, and the retention rates were calculated based on

119 homologous genes on corresponding chromosome (Voorrips, 2002).

\section{Analysis of $\mathbf{J m j C}$ sequences}

121 The gene structures were visualized by GSDS (http://gsds.cbi.pku.edu.cn/). DOG program was

122 used to sketch site information of domain organization (Ren et al., 2009). Multiple sequence

123 alignment which is based on the full-length protein sequences is performed by ClustalW

124 (Thompson et al., 1994), and its resulting files were subjected to phylogenic analysis by

125 neighbor-joining method in MEGA7.0 program with pairwise deletion, p-distance model and

126 Bootstrap test of 1000 replicates (Tamura et al., 2013). The resulting files were subjected to

127 phylogenic analysis by Maximum likelihood with pairwise deletion, Nearest-Neighbor-

128 Interchange and Bootstrap test of 1000 replicates. CDS sequences of JmjC were aligned with

129 ClustalW to create Logo maps (http://weblogo.berkeley.edu/logo.cgi), and the Fe(II) binding site

130 are showed in red triangle and $\alpha \mathrm{KG}$ binding site are showed in black triangle.

\section{Duplicated JmjC Genes in B. napus}

132 The duplicated genes of B. napus were defined by the method of Yang et al (Yang et al., 2008)

133 and Sun et al (Sun et al., 2015). The CDS sequence coverage and amino acid identity were

134 determined by Blastn/Blastp. The number of non-synonymous mutations (Ka) and the number of

135 synonymous substitutions (Ks) of duplicated genes were calculated by DnaSP 6.0 (Town et al.,

136 2006). The duplication time was calculated by $\mathrm{T}=\mathrm{Ks} / 2 \lambda \times 10^{-6}\left(\lambda=1.5 \times 10^{-8}\right)$ (Koch et al.,

137 2000).

138 Plant Material and Stress Treatment

139 The Brassica napus L. ssp Xiangyou 15 was used as the plant material of stress treatment. The

140 seeds were provided by Key Laboratory of Crop Epigenetic Regulation and

141 Development in Hunan Province, Hunan Agricultural University. B. napus 
142 seedlings were grown on clay substrates at $22^{\circ} \mathrm{C}$ chamber in a $16 \mathrm{~h}$ light $/ 8 \mathrm{~h}$ dark photoperiod.

143 One-month old plants with 4 true leaves were treated. For drought stress, the seedlings were

144 grown without watering, and leaves were sampled at 0, 5, 10, and 15 days. For salt stress,

145 seedlings were treated with $0,100,200$, and $300 \mathrm{mM} \mathrm{NaCl}$, and leaves were harvested at 3 days

146 after treatment. For high temperature stress, seedlings were grown at $40{ }^{\circ} \mathrm{C}$, and leaves were

147 harvested at $0,12,24$, and $36 \mathrm{~h}$ after treatment. All harvested samples were immediately frozen

148 in liquid nitrogen. Three independent biological replicates for each treatment were conducted.

149 RNA Extraction and Real-Time Quantitative PCR (RT-qPCR)

150 Samples RNA was extracted by TRIzol reagent kit (Invitrogen, Carlsbad, CA, US) and reverse

151 transcribed into cDNA by Revert Aid RT Kit (Thermo Fisher, USA). The specific primer pairs

152 (Supplement File 4) used for real-time PCR with Fast Start Universal SYBR Green Master

153 (ROX) (Roche, Switzerland) on a CF x 96 Real Time System (BIORAD). The BnActin gene

154 (accession ID: NC_027768) was used as reference gene. Each sample was run in triplicate and

155 their expression levels were analyzed by $2{ }^{-\Delta \Delta C T}$ method (Livak \& Schmittgen, 2001).

156 RESULTS

157 Chromosome Maps of JmjC Genes in Brassica

158 In this study, 21 Arabidopsis JmjC proteins were used as queries to Blastp in Brassica genomics

159 (http://brassicadb.org/brad/). B. napus carried $65 \mathrm{JmjC}$ genes, whereas its parents B. rapa and B.

160 oleracea had 29 and 23, respectively (Supplement File 1). 57 JmjC genes of B. napus were

161 mapped on 19 chromosomes (AACC, $2 n=38$ ), and 8 genes were still on scaffolds, in which 5

162 were from $\mathrm{Cn}$ subgenome and 2 from An subgenome. In addition, 23 B. oleracea genes and $29 \mathrm{~B}$.

163 rapa genes lactated on $\mathrm{C} 01-\mathrm{C} 09$ and A01-A10 chromosomes, respectively.

164 The JmjC genes in An and Cn subgenomes of B. napus show nearly identical distributions to

165 its ancestor genomes B. rapa (A-genome, 29) and B. oleracea (C-genome, 23) (Fig. 1). A02 and 
166 A07 chromosomes only exist in one member of $B$. napus, which is similar to its ancestor $B$. rapa

167 genomes. A09 chromosome carries the highest number, seven genes. Four tandem JmjC genes

168 pairs located on chromosomes A03, A09, and C03 in B. rapa (Fig. 1). The tandem duplicated

169 genes BnJMJ27;e and BnJMJ27;f on A03 subgenome are derived from BrJMJ27;a and

170 BrJMJ27;b, which belong to AtJMJ27 orthology. Tandem duplicated gene pairs

171 BnJMJ27;d/BnJMJ27;b and BnJMJ17;a/BnJMJ17;c might have resulted from the forming

172 processes allotetraploidy of B. napus. However, BnJMJ27; $a$ and BnJMJ27;g of C03 subgenome

173 are absent from the ancestor $B$. oleracea genomes, and the orthologous genes of these tandemly

174 duplicated genes appear in the corresponding location of A03 subgenome, which indicate that

175 BnJMJ27; $a$ and BnJMJ27; $g$ might have derived from the cross duplication of A03 subgenome.

176 BnJMJ31; $a$ of C03 subgenome, BnJMJ18;c of C08 subgenome, BnJMJ17;b, and BnJMJ29;c of

177 C09 subgenome may have similar origins.

178 Phylogenetic Analysis of JmjC Proteins in B. napus

179 A phylogenetic tree was constructed with $21 \mathrm{JmjC}$ proteins form Arabidopsis, 19 from O. sativa,

18029 from B. rapa, 23 from B. oleracea, and 65 from B. napus to examine their relationships. To

181 make the name of JmjC gene more coherent and rational, JmjC genes of Brassica were named

182 based on their relationship to homologous gene in Arabidopsis. The NJ tree that has similar

183 topology with ML tree and more high bootstrap values, is used to analyze the phylogenetic

184 relationship of JmjC proteins (Fig. 2; Supplement file 3). The JmjC proteins of Brassica were

185 divided into seven clades, except BoJMJ19;c and BoJMJ19;d: KDM4/JHDM3, KDM5A and B,

186 JmjC domain only A and B, JMJD6 and KDM3/JHDM2 groups. This classification pattern was

187 similar to the one previously reported for JmjC-domain proteins in the green lineage (Huang et 188 al., 2016). JmjC, Jumonji $\mathrm{N}(\mathrm{JmjN})$ and zinc-finger (ZnF) motifs were the special motifs for

189 KDM4/JHDM3; JmjC, JmjN, F/Y-rich N terminus (FYRN) and F/Y-rich C terminus (FYRC) for 
190 KDM5A; JmjC, JmjN and plant homeodomain (PHD) for KDM5B; JmjC and F-box for JMJD6;

191 JmjC and RING (really interesting new gene) for KDM3/JHDM2; and JmjC domain for JmjC

192 domain only A/B (Figs. 3-7). JmjN domain specifically exists in all proteins of KDM4/JHDM3,

193 KDM5A and KDM5B group, except BrJMJ14;a, BnJMJ16;b, BrJMJ17 and BnJMJ17;c. B. rapa,

194 Arabidopsis and O. sativa possess similar amounts of JmjC proteins in KDM5B, JmjC domain-

195 only A, JMJD6 and JmjC domain only B group. However, B. oleracea does not have JmjC

196 protein of KDM5B and JmjC domain-only A. B. napus has $63 \mathrm{JmjC}$ proteins, which is more than

197 the sum of those for B. oleracea and B. rapa (Fig. 2; Supplement File 1). The gene pairs imply

198 the closest relatives within the phylogenetic tree. JmjC phylogenetic tree identified 39 sister pairs

199 consisting of $22 \mathrm{An}-\mathrm{Ar}$ and $17 \mathrm{Cn}-\mathrm{Co}$ (Fig. 2). Moreover, most of the sister pairs are also

200 paralogous gene pairs between the An and Cn subgenomes (Fig. 2).

\section{Group KDM4/JHDM3}

202 Group-KDM4/JHDM3 contains nine JmjC proteins from B. napus, four from B. rapa, five from

203 B. oleracea, five from O. sativa and three from Arabidopsis (Fig. 3). Group-KDM4/JHDM3 can

204 be divided into two subgroups according to phylogenetic relationship, domain characteristic and

205 gene structure: subgroup-I with eight Brassica members and two Arabidopsis homologous genes,

206 AtJMJ11 and AtJMJ12.

207 The domain organization of subgroup-I members show highly-conserved and shared JmjC,

208 JmjN and ZnF domain. Subgroup-II contains 10 Brassica members and Arabidopsis homologous

209 gene AtJMJ13 shared JmjC, and JmjN (Figs. 3A and 3B). KDM4 subfamily shares the JmjN and

210 JmjC motifs. JmjN domain is the second highly-conserved domain that is close to the $\mathrm{N}$ terminus

211 and shorter than JmjC domain (Balciunas \& Ronne, 2000). The four tandem array ZnF domain of

212 RELATIVE OF EARLY FLOWERING 6 (REF6)/AtJMJ12 targets motif CTCTGYTY, and the

$213 \mathrm{ZnF}$ domain only exists in subgroup-I ( $\mathrm{Li}$ et al., 2016). REF6 also tends to bind to hypo- 
214 methylated CTCTGYTY motifs in vivo (Qiu et al., 2019). Subgroup-I generally harbors 7-8

215 exons, but subgroup-II keeps highly similar gene structures with 10 exons (Fig. 3C).

216 JmjC proteins have been discovered as Fe(II)- and $\alpha \mathrm{KG}$-dependent histone demethylases

217 (Chen et al., 2006; Klose et al., 2006). The JmjN and JmjC domains, two non-adjacent domains,

218 interact with each other through two $\beta$-sheets and form a single functional unit to ensure the

219 stability and appropriate transcription activity of Gis1 and maintain the overall protein levels and

220 function of Jhd2 H3K4-specific demethylase in budding yeast (Huang et al., 2010; Quan et al.,

221 2011). KDM4/JHDM3 has conserved Fe(II) binding site (His and Gluresidues) and $\alpha \mathrm{KG}$ binding

222 site (Phe and Lysresidues) (Fig. 3D).

223 Group-KDM5A/B

224 KDM5/JARID1 further can be divided into two groups: KDM5A and KDM5B (Fig. 2;

225 Supplement File 2). Group-KDM5A contains $18 \mathrm{JmjC}$ proteins from B. napus, 2 from O. sativa,

22610 from B. rapa, 7 from B. oleracea and 5 from Arabidopsis (Fig. 4A). Group-KDM5B only has

2274 JmjC proteins from Brassica and 1 from Arabidopsis homologous gene AtJMJ17. B. oleracea

228 does not have KDM5B JmjC proteins, and B. napus carries 3 members.

229 Group-KDM5A is distinguished by JmjC, JmjN, FYRN and FYRC motifs (Fig. 4B), 230 and.can be further divided into three subgroups: subgroup-I with 18 Brassica members and 3 231 Arabidopsis homologous genes, AtJMJ14, AtJMJ15 and AtJMJ18. These members show highly232 conserved domain organization sharing $\operatorname{JmjC}, \operatorname{JmjN}, \mathrm{FYRN}$, and FYRC domains, except 233 BrJMJ14; . The phylogenetic tree showed that B. oleracea does not have AtJMJ18 homologues.

234 Moreover, BrJMJ18; $b$ is clustered with BnJMJ18; $a$ and BnJMJ18; $b$, as well as BrJMJ18; $a$ with 235 BnJMJ18; and BnJMJ18;d (Fig. 4A). However, B. napus does not have a gene clustered with 236 BrJMJ14;b, BoJMJ15; b, and BrJMJ15;b. Subgroup-II has seven Brassica members and 237 Arabidopsis homologous gene AtJMJ16 sharing JmjC, FYRN, JmjN and FYRC domains, except 
238 BnJMJ16;e, which display highly-conserved domain organization, in addition to BrJMJ16; $a$ with

239 additional helicase superfamily C-terminal and DEAD-like helicases superfamily domains.

240 Subgroup-III has 10 Brassica members and Arabidopsis homologous genes AtJMJ19 and share

$241 \mathrm{JmjC}$ and JmjN domains, besides BnJMJ19; a and BnJMJ19; b with an additional transmembrane

242 domain. This finding suggested that subgroup-III may have a relatively stable inheritance during

243 the formation process of allotetraploidy.

244 Group-KDM5B differs from group-KDM5A group in domain organization, which has

245 BRIGHT and PHD but lacking FYRN and FYRC (Fig. 4F). BRIGHT is associated with H3K4

246 demethylase by DNA binding motif (CCGCCC) to regulate transcription (Tu et al., 2008). PHD

247 mainly exerts epigenetic effectors capable of recognizing or "reading" post-translational histone

248 modifications and unmodified histone tails (Musselman \& Kutateladze, 2011). The original PHD

249 role in gene transcription is acted as a reader of H3K4me3 in 2006 (Wysocka et al., 2006). Many

250 sophisticated functions of PHD were also determined, including H3K9me3 recognition and

251 binding to the $\mathrm{N}$-terminus of $\mathrm{H} 3$, indicating its key roles in regulating transcription and chromatin

252 structure (Wang et al., 2015). All members of group-KDM5B group have BRIGHT or PHD

253 domains (Fig. 4F), indicating their involvement in demethylation using JmjC domain associated

254 with BRIGHT and PHD domains.

255 Group-KDM5A/B shows a wide range intron/exon number (5-36), but sister gene pairs are

256 relatively conserved in gene structure (Fig. 4). In group-KDM5A, subgroups-I/II are highly

257 conserved in $\mathrm{Fe}(\mathrm{II})$ and $\alpha \mathrm{KG}$ binding sites, except BrJMJ16;b in which Phe is replaced by Met in

$258 \alpha \mathrm{KG}$ binding site, and His is replaced by Arg Fe(II) binding site. In subgroup-III, Phe is replaced

259 by Gln in $\alpha \mathrm{KG}$ binding site, and BoJMJ19;c/d is variable in other $\mathrm{Fe}(\mathrm{II})$ and $\alpha \mathrm{KG}$ binding sites

260 (Fig. 4D). In group-KDM5B, BnJMJ17a gene structure is similar to its parent BrJMJ17 (Fig. 
261 4G). Group-KDM5B is highly conserved in $\mathrm{Fe}(\mathrm{II})$ and $\alpha \mathrm{KG}$ binding sites, similar to

262 KDM4/JHDM3 group (Fig. 4H).

\section{Group-JmjC Domain-only A/B}

264 Group-JmjC domain-only A/B and JMJD6 are distributed in different branches of a large clade.

265 Group-JmjC domain-only A is close to group-JMJD6 but far from group-JmjC domain-only B.

266 Group-JmjC domain-only A and B have same domain organization and only exist in JmjC 267 domain (Fig. 2).

268 Group-JmjC domain-only A possesses the least number of JmjC proteins among the groups 269 (Figs. 2 and 5A) and contains three Brassica members and one Arabidopsis homologous gene 270 AtJMJ20. B. oleraace is lack of Group-JmjC domain-only A JmjC proteins (Fig. 5). BnJMJ20;b

271 shares coincident gene structures, domain organizations and chromosomal map with BrJMJ20

272 (Figs. 1, 5B and 5C) indicating that the former may have originated from the latter. Chromosomal 273 map, CDS cover and protein ID reveal that BnJMJ20;a might be the duplicate of BnJMJ20;b 274 (Supplement File 2).

275 JmjC domain-only B contains 17 JmjC proteins: 6 from B. napus, 2 from B. oleracea, 3 276 from B. rapa, 1 from O. sativa and 3 from Arabidopsis. Group-JmjC domain-only B can be 277 further divided into three subgroups. Subgroup-I contains four Brassica members and 278 Arabidopsis homologous gene AtJMJ30 (Fig. 5E). Subgroup-II contains three Brassica members 279 and Arabidopsis homologous gene AtJMJ31. Subgroup-III contains four Brassica members and 280 Arabidopsis homologous gene AtJMJ32 (Fig. 5E). Subgroups-I and III show high conservation 281 during the forming process of allotetraploid. B. napus perfect inherited JmjC genes from its 282 parents B. oleracea and B.rapa: BnJMJ30;b originating from BoJMJ30;a and BnJMJ30;a from 283 BrJMJ30;a within subgroup-I; BnJMJ32;b originating from BoJMJ32;a and BnJMJ32;a from

284 BrJMJ32 in subgroup-III. B. oleracea lacks JmjC proteins in subgroup-II. BnJMJ31;a exhibits 
285 notable similarity with BnJMJ31;b in terms of domain component and gene structure, indicating

286 that BnJMJ31;a may have originated from the inserted duplicate of BnJMJ31;b belonged to

287 paralogues gene (Figs. 5F and 5G).

288 Group-JmjC domain-only A has stable exon distribution harboring approximately 7-9

289 exons. Sequence alignment and logos analysis of JmjC domain reveal that JmjC domain-only A

290 group is highly conserved in $\mathrm{Fe}(\mathrm{II})$ and $\alpha \mathrm{KG}$ binding sites. However, compared with that in the

291 KDM4/JHDM3 group, Phe is replaced by Thr in Fe(II) binding site (Fig. 5D). In group-JmjC

292 domain-only B, subgroup-I genes contains 6 exons, subgroup- III harbors 4 exons, and subgroup-

293 II has many exons (Fig. 5G). As compared with that in the KDM4/JHDM3 group, the Phe residue

294 is replaced by Ser within AtJMJ31 orthology (Fig. 5H).

\section{Group-JMJD6}

296 The phylogenetic tree showed that the JMJD6 group is close to JmjC domain-only A group and

297 includes five JmjC proteins from B. napus, three from B. oleracea, two from Arabidopsis, two

298 from B. rapa and two from O. sativa. Each JmjC gene of B. napus is clustered with a

299 corresponding homologous gene from B. oleracea or B. rapa (Figs. 1 and 6A).

300 On the basis of phylogenetic tree analysis and schematic diagrams, group-JMJD6 can be

301 further divided into two subgroups (Fig. 6). Subgroup-I contains six Brassica members and

302 Arabidopsis homologous gene AtJMJ21 having only JmjC domain, besides BoJMJ21;b protein

303 with an additional F-box domain (Figs. 6A and 6B). Subgroup-II contains four Brassica members

304 and AtJMJ22, which shares JmjC and F-box domains except AtJMJ22 missing F-box domain.

305 However, their gene structure shows high conservation (Fig. 6). F-box domain recognizes a wide

306 array of substrates and regulates many important biological processes by degrading cellular

307 proteins in plants (Gupta et al., 2015). 
Subgroup-I generally harbors 15-16 exons, except BoJMJ21;a (4 exon) and BnJMJ21;a (9 with that in KDM4/JHDM3 group, Phe is replaced by Ala within AtJMJ21 orthology and by Ser

311 within AtJMJ22 orthology in JMJD6 (Fig. 6D).

\section{Group-KDM3/JHDM2}

313 The KDM3 \& JHDM2 group is the largest group with 48 JmjC proteins: 6 from Arabidopsis 314 (JMJ24-29), 22 from B. napus, 8 from B. rapa, 5 from O. sativa, and 6 from B. oleracea (Figs. 2 315 and 7A). Group-KDM3 \& JHDM2 can be divided into four subgroups: subgroup-I containing 14 316 Brassica members and 3 Arabidopsis homologous genes, AtJMJ25, AtJMJ26 and AtJMJ29.

317 These proteins have AT-hook motif, RING and DM domains, except JmjC domain. Subgroups318 II/III/IV contain AtJMJ27, AtJMJ24 and AtJMJ28 and their homologue genes, respectively. 319 Subgroups-III/IV show highly-conserved and shared JmjC and RING domains, except 320 BoJMJ24;a (Fig. 7B). Moreover, their gene structure also shows corresponding conservation 321 (Fig. 7C).

322 In group-KDM3/JHDM2 (Fig. 7B), half of the members harbor RING domain as the second 323 primary domain. Cys- $\mathrm{X}_{2}-\mathrm{Cys}-\mathrm{X}_{(9-39)}-\mathrm{Cys}-\mathrm{X}_{(1-3)}-\mathrm{His}-\mathrm{X}_{(2-3)}-\mathrm{Cys}-\mathrm{X} 2-\mathrm{Cys}-\mathrm{X}_{(4-48)}-\mathrm{Cys}-\mathrm{X}_{2}-\mathrm{Cys}$ is the 324 canonical RING (Deshaies and Joazeiro, 2009). The RING domain of many proteins mainly

325 binds to ubiquitin-conjugating enzymes and mediates the direct transfer of ubiquitin to substrate

326 (Deshaies \& Joazeiro, 2009). The AT-hook is a small DNA-binding motif with a preference for

327 A/T rich regions found in various proteins, such as the high mobility group proteins (Klosterman 328 and Hadwiger, 2002).

329 Sequence alignment and logos analysis of the JmjC domain reveal that subgroups-I and II 330 are highly conserved in Fe(II) binding sites (His, Asp and Cys) and $\alpha \mathrm{KG}$ binding sites (Thr and 331 Lys), except BoJMJ29;a. Moreover, both sites of subgroup-IV are different: The His and Asp 
332 residues of $\mathrm{Fe}(\mathrm{II})$ binding sites are replaced by Gly and Glu residues, and the Thr of $\alpha \mathrm{KG}$ binding

333 sites is replaced by Lys residue (Fig. 7D). However, subgroup-III does not present conservation.

\section{Stress-response expression of KDM5 subfamily genes}

335 Arabidopsis KDM5 subfamily genes play central roles in stress-responsive gene expression and

336 gene priming by H3K4me3 demethylation (Jaskiewicz et al., 2011). The expression of genes

337 related to the response for drought, high temperature and saline stresses was determined to

338 characterize the corresponding function of KDM5 group homologues in B. napus abiotic stress

339 response.

340 Under three different stress conditions, the expression profiles of BnKDM5 subfamily genes

341 were detected by real-time PCR (Fig. 8-10). BnJMJ16;a, BnJMJ17;b/c and BnJMJ18; $a$ showed

342 remarkably elevated expression under salt, drought and high temperature. However, BrJMJ19;a/c

343 did not show significant expression changes. The vast majority of JmjC genes showed

344 remarkably elevated expression under drought treatment, except BnJMJ14; $a$ and BnJMJ19;a/c/e.

345 Under drought 5 or 10 day, most of the genes had higher expression than drought 15, except

346 BnJMJ17;c and BnJMJ19;b (Fig. 8). However, only 6 (BnJMJ16; $a, B n J M J 17, B n J M J 18 ; a$ and

347 BnJMJ19;e) out of the $20 \mathrm{JmjC}$ genes showed elevated expression under high temperature

348 treatment. BnJMJ16; $a, B n J M J 17 a / b$ and $B n J M J 18$; $a$ expression was induced under 12 hours of

349 high temperature treatment, but BnJMJ17;c and BnJMJ19;e were not substantially expressed

350 until 36 hours (Fig. 9). Moreover, nearly half of the JmjC genes (BnJMJ14, BnJMJ15; a,

351 BnJMJ16;a, BnJMJ17;b/c and BnJMJ18;a/d) showed remarkable expression under $100 \mathrm{Mm}$

$352 \mathrm{NaCl}$ treatment, besides BnJMJ15;c that was strongly induced by $200 \mathrm{mM} \mathrm{NaCl}$ stress ( Fig. 10).

353 DISCUSSION

354 Conserved JmjC Genes of B. napus 
355 Allotetraploid species B. napus (AACC, 2n $=38$ ) derived from interspecific spontaneous 356 hybridization of $B$. rapa (AA, $2 \mathrm{n}=20)$ and $B$. oleracea $(\mathrm{CC}, 2 \mathrm{n}=18)$ (Nagaharu, 1935). Nuclear

357 genomes have remained essentially unaltered since amphidiploid species formation (Parkin et al., 358 1995). Similarly, the JmjC protein family appears to be extremely conserved during B. napus

359 formation. Compared with the progenitor genomes of B. rapa and B. oleracea, 27 (93.1\%) JmjC 360 orthologous gene pairs between An subgenome and $19(82.6 \%)$ between $\mathrm{Cn}$ subgenome in $B$. 361 napus were conserved (Fig. 2). The average retention rates from ancestor exceed the rate of all 362 homologous gene pairs (83.7\%) across the whole B. napus genome (Chalhoub et al., 2014). Each 363 member of B. rapa and B. oleracea can be paired to at least one homologue of B. napus, except 364 five members of KDM5A subfamily: BrJMJ14;b, BrJMJ15;b, BoJMJ15;b, BoJMJ19;c and $365 B o J M J 19 ; d$, which indicates the JmjC genes are highly conserved but some reductions might 366 have been found in KDM5A subfamily during the formation process of allotetraploid (Fig. 4). 367 Comparing with the reported homologous genes, BrJMJ14;b, BrJMJ15; $b$ and BoJMJ15; $b$ might 368 be associated with floral integrators and flowering time by H3K4 demethylase activities (Lu et 369 al., 2010; Yang et al., 2010; Yang et al., 2012b). However, the BrJMJ14;b, BrJMJ15;b and 370 BoJMJ15;b might be redundant, because their paralogs can be found in B. napus (Fig. 4). In 371 addition, BoJMJ19;c and BoJMJ19;d have present difference in structures and might only 372 specifically exist in B. oleracea (Fig. 4).

373 Gene duplication expands genome content and changes gene function to ensure the optimal 374 adaptability and evolution of plants (Xu et al., 2012). The $65 \mathrm{JmjC}$ proteins from B. napus were 375 more than the total number of proteins for B. rapa (29) and B. oleracea (23) (Supplement File 1). 376 According to the systematic analysis results of JmjC proteins, some new or duplicated JmjC 377 genes were found in B. napus (Figs. 2-7). Gene duplication events were confirmed by the method 378 of Yang et al. (Yang et al., 2008) and Sun et al. (Sun et al., 2015). BnJMJ16;e/BnJMJ16;d, 
381 derived from the existing JmjC gene from B. rapa and BnJMJ28;a/BnJMJ28; $b$ and BnJMJ29;a/

382 BnJMJ29;c pairs from B. oleracea (Supplement File 1). These gene pairs were duplicated

383 through segmental duplication (Supplement File 2). Additionally, the parent of BnJMJ17;c was

384 not found by the method, but its sequence of JmjC domain was consistent with the BnJMJ17; $b$.

385 In crop species, gene duplicate events can contribute to the evolution of novel functions and

386 important agronomic traits, such as fruit shape, flowering time, disease resistance and adaptation

387 to stress (Panchy et al. 2016). In contrast, whole genome triplication event of Brassica rapa and

388 Brassica oleracea exerts critical roles in the speciation and morphotype diversification of

389 Brassica plants (Cheng et al. 2014).

390 Overall, the JmjC genes of B. napus were conserved during the formation process of

391 allotetraploidy, and the gene reduction and duplication from parents were preferred in the 392 KMD5A group.

\section{Conservation and Function of JmjC Proteins of B. napus}

$39465 \mathrm{JmjC}$ proteins of B. napus were clustered into seven groups based on phylogenetic and domain 395 organization (Figs. 2-7; Supplement File 1) similar to the result that JmjC domain proteins is 396 systematic analyzed ranging from green alga to higher plant (Huang et al., 2016). Furthermore, 397 the BnJmjC demonstrated high similarity with homologous sequences or even with the whole 398 subfamily in domain origination, chromosomal location, intron/exon number and catalytic sites.

399 These results indicated JmjC proteins of B. napus were conserved family during allotetraploid 400 formation.

401 In general, H3K4me2/me3 and H3K36 correlate with transcriptional activation, and 402 H3K9me2 and H3K27me3 correlate with gene silencing (Huang et al., 2011). The substrate 
403 specificity of BnJmjC proteins can be predicted based on their conservation and previous

404 research results. KDM4/JHDM3 was involved in multi-demethylation (H3K4me2/3, H3K9me3,

$405 \mathrm{H} 3 \mathrm{~K} 27 \mathrm{~m} 2 / 3$ and H3K36me2/3), such as AtJMJ11 for H3K27m3, H3K9me3 and H3K4me3

406 demethylation (Bendahmane et al., 2009; Crevillen et al., 2014; Noh et al., 2004; Yu et al., 2008),

407 AtJMJ12/REF6 for H3K4me2/3, H3K27me2/3 and H3K36me2/3 demethylation (Cui et al.,

408 2016; Hou et al., 2014; Hyun et al., 2016; Ko et al., 2010; Li et al., 2016; Lu, F. et al., 2011) and

409 OsJM12;a/JMJ705 for H3K27me2/3 demethylation (Li et al., 2013). KDM5A was involved in

$410 \mathrm{H} 3 \mathrm{~K} 4 \mathrm{me} 2 / 3$ demethylase activity, and the activity has been reported in AtJMJ14/15/18 and

411 JMJ703(OsJM16;a)/ JMJ704/OsJM14;a (Lu et al., 2010; Yang et al., 2010; Yang et al., 2012b;

412 Shen et al., 2014; Yang et al., 2012a; Chen et al., 2013; Cui et al., 2013; Hou et al., 2015). JmjC

413 domain-only B is involved in H3K36me2 and H3K27me3 demethylation, but only a member

414 (AtJMJ30) is identified in this subfamily (Yan et al., 2014; Gan et al., 2014). KDM3/JHDM2 is

415 involved in H3K9 demethylation, and the demethylase activity has been reported in AtJMJ25/27

416 (Dutta et al., 2017; Saze et al., 2014). KDM5B might be associated with H3K4 demethylase by

417 BRIGHT and PHD domains, but there are still no reports on the members of this family.

418 Moreover, JmjC domain-only A subfamily member AtJMJ20 has a crucial role in removing

419 histone arginine methylases (Cho et al., 2012). JMJD6 subfamily member AtJMJ22 acts as

420 histone arginine demethylases (Cho et al., 2012).

421 KDM5 Response to Abiotic Stresses

422 Epigenetic marks in $\mathrm{H} 3 \mathrm{~K} 4$ exert critical functions on regulating genes response to ambient stress 423 (Baulcombe \& Dean, 2014; Begcy \& Dresselhaus, 2018). In rice, H3K4 dimethylation of $A D H 1$ 424 and PDC1 is switched to trimethylation to response to submergence stress (Qiao \& Fan, 2011). $425 \mathrm{H} 3 \mathrm{~K} 4 \mathrm{me} 3$ is also correlated with gene expression which responds to dehydration stress (Santos et 426 al., 2011). Arabidopsis H3K4me3 of AHG3, catalyzed by ATX4 and ATX5, plays an essential 
427 role in drought stress response (Liu, Y. et al., 2018). Arabidopsis H3K4 hypermethylation is

428 associated with transcriptional activation and maintenance heat stress response (Liu, H.C. et al.,

429 2018). Furthermore, Over-expression of KDM5 subfamily AtJMJ15, a H3K4 demethylase,

430 enhanced salt tolerance (Shen et al., 2014). In KDM5 subfamily, the similar gene sequences and

431 domain organization between Arabidopsis and B. napus suggests that B. napus members may

432 also possess conserved biological function with $\mathrm{H} 3 \mathrm{~K} 4$ demethylase activity (Fig. 4). The

433 expression patterns of BnKDM5 subfamily show that almost all of BnKDM5 genes are involved

434 in drought, high temperature and salt stress response (Fig. 8-10).

435 Under drought, high temperature or salt stress, all members of BnKDM5B exhibited 436 remarkable elevated expression, except BnJMJ17;a under salt stress, suggesting that these 437 homologous genes have conserved functions to responses to similar stress stimuli. In general, the 438 expression of $B n K D M 5 A$ genes are relatively conserved to response to identical stress condition.

439 For instance, the homologous gene of AtJMJ15 (BnJMJ15;a/b/c), AtJMJ16 (BnJMJ16;a/b/c/d), 440 and AtJMJ18 (BnJMJ18;a/b/c/d) showed similar stress response to drought stress with 441 remarkable increased expression. However, their display diverse transcriptional responses to 442 other stress stimuli, even among homologous genes. For example, BnJMJ18; $a$ shows remarkably 443 elevated expression under high temperature without the homologous genes $B n J M J 18 ; b / c / d$. 444 These results indicate that functions of BnKDM5 members are conserved and divergent during 445 allotetraploid formation.

\section{Conclusions}

447 This study provides the first genome-wide characterization of JmjC genes in Brassica species. The BnJmjC 448 exhibits higher conservation during the formation process of allotetraploid than the average retention rates of 449 whole B. napus genome. Furthermore, expression profiles indicated that BnKDM5 subfamily genes are 450 involved in stress response to salt, drought and high temperature.

\section{ACKNOWLEDGEMENTS}


452 We are grateful to Brassica database (http://brassicadb.org/brad/) and its contributors who provided their data 453 for this analysis.

454

455

456

457

458

459

460

461

462

463

464

465

466

467

468

469 REFERENCES

470 Balciunas D, Ronne H. 2000. Evidence of domain swapping within the jumonji family of transcription factors.

471 Trends in biochemical sciences 25:274-276 DOI 10.1016/s0968-0004(00)01593-0

472 Bannister AJ, Kouzarides T. 2011. Regulation of chromatin by histone modifications. Cell Research 21:381-395

473 DOI 10.1038/cr.2011.22

474 Baulcombe DC, Dean C. 2014. Epigenetic regulation in plant responses to the environment. Cold Spring

475 Harbor Perspectives in Biology 6(9): a019471 DOI 10.1101/cshperspect.a019471 
476 Begcy K, Dresselhaus T. 2018. Epigenetic responses to abiotic stresses during reproductive development in 477 cereals. Plant Reprod 31:343-355 DOI 10.1007/s00497-018-0343-4

478 Bej S, Basak J. 2017. Abiotic Stress Induced Epigenetic Modifications in Plants: How Much Do We Know?. In: 479 Rajewsky N, Jurga S, Barciszewski J. (eds) Plant Epigenetics. RNA Technologies. Springer, Cham DOI 480 10.1007/978-3-319-55520-1_24

481 Chalhoub B, Denoeud F, Liu S, Parkin IA, Tang H, Wang X, Chiquet J, Belcram H, Tong C, Samans B, Correa 482 M, Da Silva C, Just J, Falentin C, Koh CS, Le Clainche I, Bernard, M., Bento, P., Noel, B., Labadie, K., Alberti, 483 A., Charles, M., Arnaud, D., Guo H, Daviaud C, Alamery S, Jabbari K, Zhao M, Edger PP, Chelaifa H, Tack D, 484 Lassalle G, Mestiri I, Schnel N, Le Paslier MC, Fan G, Renault V, Bayer PE, Golicz AA, Manoli S, Lee TH, 485 Thi VH, Chalabi S, Hu Q, Fan C, Tollenaere R, Lu Y, Battail C, Shen J, Sidebottom CH, Wang X, Canaguier A, 486 Chauveau A, Berard A, Deniot G, Guan M, Liu Z, Sun F, Lim YP, Lyons E, Town CD, Bancroft I, Wang X, 487 Meng J, Ma J, Pires JC, King GJ, Brunel D, Delourme R, Renard M, Aury JM, Adams KL, Batley J, Snowdon 488 RJ, Tost J, Edwards D, Zhou Y, Hua W, Sharpe AG, Paterson AH, Guan C, Wincker P. 2014. Plant genetics. 489 Early allopolyploid evolution in the post-Neolithic Brassica napus oilseed genome. Science 345:950-953 DOI $490 \quad 10.1126 /$ science. 1253435

491 Chen Q, Chen X, Wang Q, Zhang F, Lou Z, Zhang Q, Zhou DX. 2013. Structural basis of a histone H3 lysine 4 492 demethylase required for stem elongation in rice. PLoS Genetics 9:e1003239 DOI 493 10.1371/journal.pgen.1003239

494 Chen Z, Zang J, Whetstine J, Hong X, Davrazou F, Kutateladze TG, Simpson M, Mao Q, Pan CH, Dai S, 495 Hagman J, Hansen K, Shi Y, Zhang G. 2006. Structural insights into histone demethylation by JMJD2 family 496 members. Cell 125:691-702 DOI 10.1016/j.cell.2006.04.024

497 Cheng F, Wu J, and Wang X. 2014. Genome triplication drove the diversification of Brassica plants. 498 Horticulture Research 1:14024 DOI 10.1038/hortres.2014.24

499 Cho JN, Ryu JY, Jeong YM, Park J, Song JJ, Amasino RM, Noh B, Noh YS. 2012. Control of seed germination 500 by light-induced histone arginine demethylation activity. Developmental Cell 22:736-748 DOI

501 10.1016/j.devcel.2012.01.024

502 Crevillén P, Yang H, Cui X, Greeff C, Trick M, Qiu Q, Cao X, Dean C. 2014. Epigenetic reprogramming that 503 prevents transgenerational inheritance of the vernalized state. Nature 515:587-590 DOI 10.1038/nature13722 
504 Cui X, Jin P, Cui X, Gu L, Lu Z, Xue Y, Wei L, Qi J, Song X, Luo M, An G, Cao X. 2013. Control of

505 transposon activity by a histone H3K4 demethylase in rice. Proceedings of the National Academy of Sciences of

506 the United States of America 110:1953-1958 DOI 10.1073/pnas.1217020110

507 Cui X, Lu F, Qiu Q, Zhou B, Gu L, Zhang S, Kang Y, Cui X, Ma X, Yao Q, Ma J, Zhang X, Cao X. 2016.

508 REF6 recognizes a specific DNA sequence to demethylate H3K27me3 and regulate organ boundary formation

509 in Arabidopsis. Nature Genetics 48:694-699 DOI 10.1038/ng.3556

510 Deshaies RJ, Joazeiro CA. 2009. RING domain E3 ubiquitin ligases. Annual Review of Biochemistry 78:399-

511434 DOI 10.1146/annurev.biochem.78.101807.093809

512 Ding Y, Avramova Z, Fromm M. 2011. The Arabidopsis trithorax-like factor ATX1 functions in dehydration

513 stress responses via ABA-dependent and ABA-independent pathways. The Plant Journal 66:735-744 DOI

514 10.1111/j.1365-313X.2011.04534.x

515 Dutta A, Choudhary P, Caruana J, Raina R. 2017. JMJ27, an Arabidopsis H3K9 histone demethylase, modulates

516 defense against Pseudomonas syringae and flowering time. The Plant Journal 91:1015-1028 DOI

$517 \quad 10.1111 /$ tpj.13623

518 Gan ES, Xu Y, Wong JY, Goh JG, Sun B, Wee WY, Huang J, Ito T. 2014. Jumonji demethylases moderate

519 precocious flowering at elevated temperature via regulation of FLC in Arabidopsis. Nature Communications

520 5:5098 DOI 10.1038/ncomms6098

521 García-Alai MM, Allen MD, Joerger AC, Bycroft M. 2010. The structure of the FYR domain of transforming

522 growth factor beta regulator 1. Protein Science 19:1432-1438 DOI 10.1002/pro.404

523 Greer EL, Shi Y. 2012. Histone methylation: a dynamic mark in health, disease and inheritance. Nature Reviews

524 Genetics 13:343-357 DOI 10.1038/nrg3173

525 Gupta S, Garg V, Kant C, Bhatia S. 2015. Genome-wide survey and expression analysis of F-box genes in

526 chickpea. BMC Genomics 16:67 DOI 10.1186/s12864-015-1293-y

527 He C, Cole P. 2015. Introduction: epigenetics. Chem Rev 115, 2223-2224 DOI 10.1021/acs.chemrev.5b00137

528 Hou X, Zhou J, Liu C, Liu L, Shen L, Yu H. 2014. Nuclear factor Y-mediated H3K27me3 demethylation of the

529 SOC1 locus orchestrates flowering responses of Arabidopsis. Nature Communications 5:4601 DOI $530 \quad 10.1038 /$ ncomms5601 
531 Hou Y, Wang L, Wang L, Liu L, Li L, Sun L, Rao Q, Zhang J, Huang S. 2015. JMJ704 positively regulates rice

532 defense response against Xanthomonas oryzae pv. oryzae infection via reducing H3K4me2/3 associated with 533 negative disease resistance regulators. BMC Plant Biology 15:286 DOI 10.1186/s12870-015-0674-3

534 Huang F, Chandrasekharan MB, Chen YC, Bhaskara S, Hiebert SW, Sun ZW. 2010. The JmjN domain of Jhd2 535 is important for its protein stability, and the plant homeodomain .PHD. finger mediates its chromatin association 536 independent of H3K4 methylation. Journal of Biological Chemistry 285:24548-24561 DOI 537 10.1074/jbc.M110.117333

538 Huang Y, Chen D, Liu C, Shen W, Ruan Y. 2016. Evolution and conservation of JmjC domain proteins in the 539 green lineage. Molecular Genetics and Genomics 291:33-49 DOI 10.1007/s00438-015-1089-4

540 Huang Y, Liu CL, Shen WH, Ruan Y. 2011. Phylogenetic analysis and classification of the Brassica rapa SET541 domain protein family. BMC Plant Biology Bio 11:175 DOI 10.1186/1471-2229-11-175

542 Hyun Y, Richter R, Vincent C, Martinez-Gallegos R, Porri A, Coupland G. 2016. Multi-layered regulation of

543 SPL15 and cooperation with SOC1 integrate endogenous flowering pathways at the Arabidopsis shoot 544 meristem. Developmental Cell 37:254-266 DOI 10.1016/j.devcel.2016.04.001

545 Jaskiewicz M, Conrath U, Peterhansel C. 2011. Chromatin modification acts as a memory for systemic acquired 546 resistance in the plant stress response. EMBO Reports 12:50-55 DOI 10.1038/embor.2010.186

547 Jiang D, Yang W, He Y, Amasino RM. 2007. Arabidopsis relatives of the human lysine-specific Demethylase1 548 repress the expression of FWA and FLOWERING LOCUS C and thus promote the floral transition. Plant Cell 549 19:2975-2987 DOI 10.1105/tpc.107.052373

550 Jeong JH, Song HR, Ko JH, Jeong YM, Kwon YE, Seol JH, Amasino RM, Noh B, Noh YS. 2009. Repression

551 of FLOWERING LOCUS T chromatin by functionally redundant histone H3 lysine 4 demethylases in 552 Arabidopsis. PLoS ONE 4:e8033 DOI 10.1371/journal.pone.0008033

553 Kim JM, To TK, Ishida J, Matsui A, Kimura H, Seki M. 2012. Transition of chromatin status during the process 554 of recovery from drought stress in Arabidopsis thaliana. Plant and Cell Physiology 53:847-856 DOI 555 10.1093/pcp/pcs053

556 Klose RJ, Kallin EM, Zhang Y. 2006. JmjC-domain-containing proteins and histone demethylation. Nature 557 Reviews Genetics 7:715-727 DOI 10.1038/nrg1945 
558 Klosterman SJ, Hadwiger LA. 2002. Plant HMG proteins bearing the AT-hook motif. Plant Sci 162:855-866

559 DOI 10.1016/S0168-9452(02)00056-0

560 Ko JH, Mitina I, Tamada Y, Hyun Y, Choi Y, Amasino RM, Noh B, Noh YS. 2010. Growth habit determination

561 by the balance of histone methylation activities in Arabidopsis. EMBO Journal 29:3208-3215 DOI

$562 \quad 10.1038 /$ emboj.2010.198

563 Koch MA, Haubold B, Mitchell-Olds T. 2000. Comparative evolutionary analysis of chalcone synthase and

564 alcohol dehydrogenase loci in Arabidopsis, Arabis, and related genera (Brassicaceae). Molecular Biology and

565 Evolution 17:1483-1498 DOI 10.1093/oxfordjournals.molbev.a026248

566 Lämke J, Brzezinka K, Altmann S, Bäurle I. 2016. A hit-and-run heat shock factor governs sustained histone

567 methylation and transcriptional stress memory. EMBO Journal 35:162-175 DOI 10.15252/embj.201592593

568 Li C, Gu L, Gao L, Chen C, Wei CQ, Qiu Q, Chien CW, Wang S, Jiang L, Ai LF, Chen CY, Yang S, Nguyen

569 V, Qi Y, Snyder MP, Burlingame AL, Kohalmi SE, Huang S, Cao X, Wang ZY, Wu K, Chen X, Cui Y. 2016.

570 Concerted genomic targeting of H3K27 demethylase REF6 and chromatin-remodeling ATPase BRM in

571 Arabidopsis. Nature Genetics 48:687-693 DOI 10.1038/ng.3555

572 Li S, Ali S, Duan X, Liu S, Du J, Liu C, Dai H, Zhou M, Zhou L, Yang L, Chu P, Li L, Bhatia R, Schones DE,

$573 \mathrm{Wu}$ X, Xu H, Hua Y, Guo Z, Yang Y, Zheng L, Shen, B. 2018. JMJD1B demethylates H4R3me2s and

$574 \mathrm{H} 3 \mathrm{~K} 9 \mathrm{me} 2$ to facilitate gene expression for development of hematopoietic stem and progenitor cells. Cell

575 Reports 23:389-403 DOI 10.1016/j.celrep.2018.03.051

576 Li T, Chen X, Zhong X, Zhao Y, Liu X, Zhou S, Cheng S, Zhou DX. 2013. Jumonji C domain protein JMJ705-

577 mediated removal of histone $\mathrm{H} 3$ lysine 27 trimethylation is involved in defense-related gene activation in rice.

578 Plant Cell 25:4725-4736 DOI 10.1105/tpc.113.118802

579 Liu C, Lu F, Cui X, Cao X. 2010. Histone methylation in higher plants. Annual Review of Plant Biology

580 61:395-420 DOI 10.1146/annurev.arplant.043008.091939

581 Liu HC, Lamke J, Lin SY, Hung MJ, Liu KM, Charng YY, Baurle I. 2018. Distinct heat shock factors and

582 chromatin modifications mediate the organ-autonomous transcriptional memory of heat stress. The Plant

583 Journal 95:401-413 DOI 10.1111/tpj.13958

Peer] reviewing PDF | (2020:02:45914:2:0:NEW 31 Jan 2021) 
584 Liu W, Tanasa B, Tyurina OV, Zhou TY, Gassmann R, Liu WT, Ohgi KA, Benner C, Garcia-Bassets I,

585 Aggarwal AK, Desai A, Dorrestein PC, Glass CK, Rosenfeld MG. 2010. PHF8 mediates histone H4 lysine 20

586 demethylation events involved in cell cycle progression. Nature 466:508-512 DOI 10.1038/nature09272

587 Liu Y, Zhang A, Yin H, Meng Q, Yu X, Huang S, Wang J, Ahmad R, Liu B, Xu ZY. 2018. Trithorax-group

588 proteins ARABIDOPSIS TRITHORAX4 (ATX4) and ATX5 function in abscisic acid and dehydration stress

589 responses. New Phytologist 217:1582-1597 DOI 10.1111/nph.14933

590 Livak KJ, Schmittgen TD. 2001. Analysis of relative gene expression data using real-time quantitative PCR and

591 the 2(-Delta Delta C(T)) method. Methods 25:402-408 DOI 10.1006/meth.2001.1262

592 Lu F, Cui X, Zhang S, Jenuwein T, Cao X. 2011. Arabidopsis REF6 is a histone H3 lysine 27 demethylase.

593 Nature Genetics 43:715-719 DOI 10.1038/ng.854

594 Lu F, Cui X, Zhang S, Liu C, Cao X. 2010. JMJ14 is an H3K4 demethylase regulating flowering time in

595 Arabidopsis. Cell Research 20:387-390 DOI 10.1038/cr.2010.27

596 Luo M, Hung FY, Yang S, Liu X, Wu K. 2013. Histone lysine demethylases and their functions in plants. Plant

597 Molecular Biology Reporter 32:558-565 DOI 10.1007/s11105-013-0673-1

598 Musselman CA, Kutateladze TG. 2011. Handpicking epigenetic marks with PHD fingers. Nucleic Acids

599 Reseaech 39:9061-9071 DOI 10.1093/nar/gkr613

600 Nagaharu U. 1935. Genome analysis in Brassica with special reference to the experimental formation of $B$.

601 Napus and peculiar mode of fertilization. Japan Journal of Botany 7:389-452

602 Noh B, Lee SH, Kim HJ, Yi G, Shin EA, Lee M, Jung KJ, Doyle MR, Amasino RM, Noh YS. 2004. Divergent

603 roles of a pair of homologous jumonji/zinc-finger-class transcription factor proteins in the regulation of

604 Arabidopsis flowering time. Plant Cell 16(10):2601-2613 DOI 10.1105/tpc.104.025353

605 Panchy N, Lehti-Shiu M, and Shiu SH. 2016. Evolution of Gene Duplication in Plants. Plant Physiology

606 171:2294-2316 DOI 10.1104/pp.16.00523

607 Parkin IA, Sharpe AG, Keith DJ, Lydiate DJ. 1995. Identification of the A and C genomes of amphidiploid

608 Brassica napus (oilseed rape). Genome 38:1122-1131

609 Qiao W, Fan L. 2011. Epigenetics, a mode for plants to respond to abiotic stresses. Frontiers in Biology 6:477-

610 481 DOI 10.1007/s11515-011-1128-4

Peer) reviewing PDF | (2020:02:45914:2:0:NEW 31 Jan 2021) 
611 Qiu Q, Mei H, Deng X, He K, Wu B, Yao Q, Zhang J, Lu F, Ma J, Cao X. 2019. DNA methylation repels

612 targeting of Arabidopsis REF6. Nature Communications 10:2063 DOI 10.1038/s41467-019-10026-1

613 Quan Z, Oliver SG, Zhang N. 2011. JmjN interacts with JmjC to ensure selective proteolysis of Gis1 by the

614 proteasome. Microbiology 157(Pt 9):2694-2701 DOI 10.1099/mic.0.048199-0

615 Ren J, Wen L, Gao X, Jin C, Xue Y, Yao X. 2009. DOG 1.0: illustrator of protein domain structures. Cell

616 Research 19:271-273 DOI 10.1038/cr.2009.6

617 Santos AP, Serra T, Figueiredo DD, Barros P, Lourenco T, Chander S, Oliveira MM, Saibo NJ. 2011.

618 Transcription regulation of abiotic stress responses in rice: a combined action of transcription factors and

619 epigenetic mechanisms. Omics A Journal of Integrative Biology 15:839-857 DOI 10.1089/omi.2011.0095

620 Saze H, Sasaki T, Kakutani T. 2014. Negative regulation of DNA methylation in plants. Epigenetics 3:122-124

621 DOI 10.4161/epi.3.3.6355

622 Shen Y, Conde ESN, Audonnet L, Servet C, Wei W, Zhou DX. 2014. Over-expression of histone H3K4

623 demethylase gene JMJ15 enhances salt tolerance in Arabidopsis. Frontiers in Plant Science 5:290 DOI 10.3389/

624 fpls.2014.00290

625 Sun R, Wang K, Guo T, Jones DC, Cobb J, Zhang B, Wang Q. 2015. Genome-wide identification of auxin

626 response factor $(A R F)$ genes and its tissue-specific prominent expression in Gossypium raimondii. Functional \&

627 Integrative Genomics 15:481-493 DOI 10.1007/s10142-015-0437-0

628 Takeuchi T, Yamazaki Y, Katoh-Fukui Y, Tsuchiya R, Kondo S, Motoyama J, Higashinakagawa T. 1995. Gene

629 trap capture of a novel mouse gene, jumonji, required for neural tube formation. Genes \& Development 9:1211-

$630 \quad 1222$

631 Tamura K, Stecher G, Peterson D, Filipski A, Kumar S. 2013. MEGA6: molecular evolutionary genetics

632 analysis version 6.0. Molecular Biology and Evolution 30:2725-2729 DOI 10.1093/molbev/mst197

633 Thompson JD, Higgins DG, Gibson TJ. 1994. CLUSTAL W: improving the sensitivity of progressive multiple

634 sequence alignment through sequence weighting, position-specific gap penalties and weight matrix choice.

635 Nucleic Acids Reseaech 22:4673-4680 DOI 10.1093/nar/22.22.4673

636 Town CD, Cheung F, Maiti R, Crabtree J, Haas BJ, Wortman JR, Hine EE, Althoff R, Arbogast TS, Tallon LJ,

637 Vigouroux M, Trick M, Bancroft I. 2006. Comparative genomics of Brassica oleracea and Arabidopsis 
638 thaliana reveal gene loss, fragmentation, and dispersal after polyploidy. Plant cell 18:1348-1359 DOI

$63910.1105 /$ tpc. 106.041665

640 Tu S, Teng YC, Yuan C, Wu YT, Chan MY, Cheng AN, Lin PH, Juan LJ, Tsai MD. 2008. The ARID domain

641 of the H3K4 demethylase RBP2 binds to a DNA CCGCCC motif. Nature Structural \& Molecular Biology

642 15:419-421 DOI 10.1038/nsmb.1400

643 Van Dijk K, Ding Y, Malkaram S, Riethoven JJ, Liu R, Yang J, Yang J, Laczko P, Chen H, Xia Y, Ladunga I,

644 Avramova Z, Fromm M. 2010. Dynamic changes in genome-wide histone H3 lysine 4 methylation patterns in

645 response to dehydration stress in Arabidopsis thaliana. BMC Plant Biology 10:238 DOI 10.1186/1471-2229-10-

646238

647 Voorrips RE. 2002. MapChart: software for the graphical presentation of linkage Maps and QTLs. Journal of 648 Heredity 93:77-78 DOI 10.1093/jhered/93.1.77

649 Wang Q, Liu J, Wang Y, Zhao Y, Jiang H, Cheng B. 2015. Systematic analysis of the maize PHD-Finger gene

650 family reveals a subfamily involved in abiotic stress response. International Journal of Molecular Sciences

$651 \quad 16: 23517-23544$ DOI 10.3390/ijms161023517

652 Wysocka J, Swigut T, Xiao H, Milne TA, Kwon SY, Landry J, Kauer M, Tackett AJ, Chait BT, Badenhorst P,

$653 \mathrm{Wu}$ C, Allis CD. 2006. A PHD finger of NURF couples histone H3 lysine 4 trimethylation with chromatin

654 remodelling. Nature 442:86-90 DOI 10.1038/nature04815

655 Xu G, Guo C, Shan H, Kong H. 2012. Divergence of duplicate genes in exon-intron structure. Proceedings of 656 the National Academy of Sciences of the United States of America 109:1187-1192 DOI 657 10.1073/pnas.1109047109

658 Yan Y, Shen L, Chen Y, Bao S, Thong Z, Yu H. 2014. A MYB-domain protein EFM mediates flowering 659 responses to environmental cues in Arabidopsis. Developmental Cell 30:437-448 DOI 660 10.1016/j.devcel.2014.07.004

661 Yang H, Han Z, Cao Y, Fan D, Li H, Mo H, Feng Y, Liu L, Wang Z, Yue Y, Cui S, Chen S, Chai J, Ma L.

662 2012a. A companion cell-dominant and developmentally regulated H3K4 demethylase controls flowering time 663 in Arabidopsis via the repression of FLC expression. PLoS Genetics 8:e1002664 DOI 664 10.1371/journal.pgen.1002664 
665 Yang H, Mo H, Fan D, Cao Y, Cui S, Ma L. 2012b. Overexpression of a histone H3K4 demethylase, JMJ15, 666 accelerates flowering time in Arabidopsis. Plant Cell Reports 31:1297-1308 DOI 10.1007/s00299-012-1249-5

667 Yang S, Zhang X, Yue JX, Tian D, Chen J Q. 2008. Recent duplications dominate NBS-encoding gene 668 expansion in two woody species. Molecular Genetics and Genomics 280:187-198 DOI 10.1007/s00438-008$6690355-0$

670 Yang W, Jiang D, Jiang J, He Y. 2010. A plant-specific histone H3 lysine 4 demethylase represses the floral 671 transition in Arabidopsis. The Plant Journal 62:663-673 DOI 10.1111/j.1365-313X.2010.04182.x

672 Yang YW, Lai KN, Tai PY, Li WH. 1999. Rates of nucleotide substitution in angiosperm mitochondrial DNA 673 sequences and dates of divergence between Brassica and other angiosperm lineages. Journal of Molecular 674 Evolution 48:597-604 DOI 10.1007/p100006502

675 Yu X, Li L, Li L, Guo M, Chory J, Yin Y. 2008. Modulation of brassinosteroid-regulated gene expression by 676 Jumonji domain-containing proteins ELF6 and REF6 in Arabidopsis. Proceedings of the National Academy of 677 Sciences of the United States of America 105:7618-7623 DOI 10.1073/pnas.0802254105

678 Zhang S, Zhou B, Kang Y, Cui X, Liu A, Deleris A, Greenberg MV, Cui X, Qiu Q, Lu F, Wohlschlegel JA, 679 Jacobsen SE, Cao X. 2015. C-terminal domains of a histone demethylase interact with a pair of transcription 680 factors and mediate specific chromatin association. Cell Discovery. 1:15003 DOI 10.1038/celldisc.2015.3

681 Zong W, Zhong X, You J, Xiong L. 2013. Genome-wide profiling of histone H3K4-tri-methylation and gene 682 expression in rice under drought stress. Plant Molecular Biology 81:175-188 DOI 10.1007/s11103-012-9990-2 683 684 685 686 687 688 689 


\section{Figure 1}

Fig. 1 Chromosomal distribution of Brassica genes

Brassica genes (57 B. napus, 23 B. oleracea and 29 B. rapa) was mapped on chromosomes except eight scaffolds genes of $B$. napus: A. B. napus genes distribution of A-genomics, B. $B$. rapa genes distribution, $C$. $B$. napus genes distribution of $C$-genomics, $D$. $B$. oleracea genes distribution. The scale on the chromosome represents megabases (Mb). 


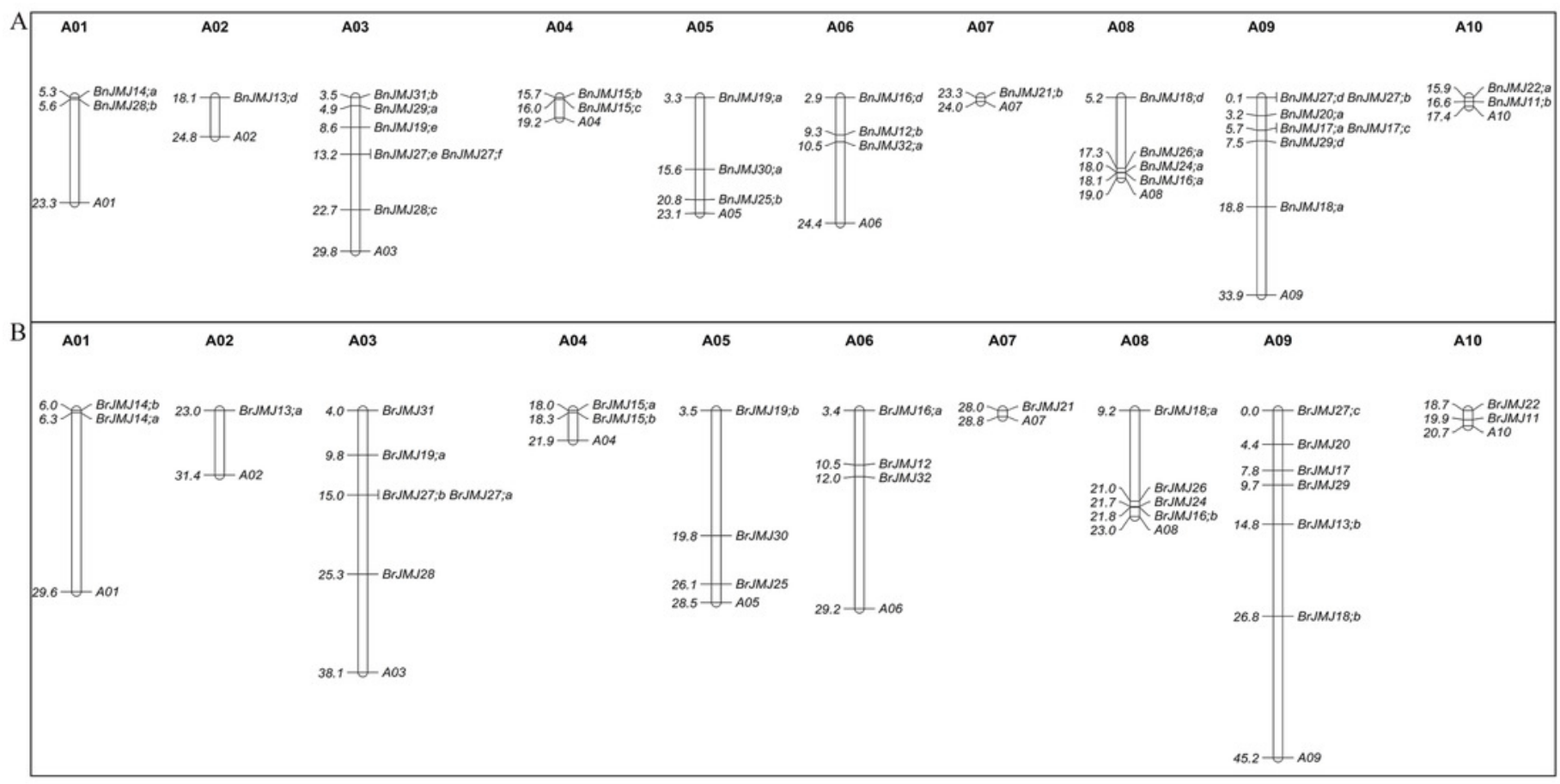

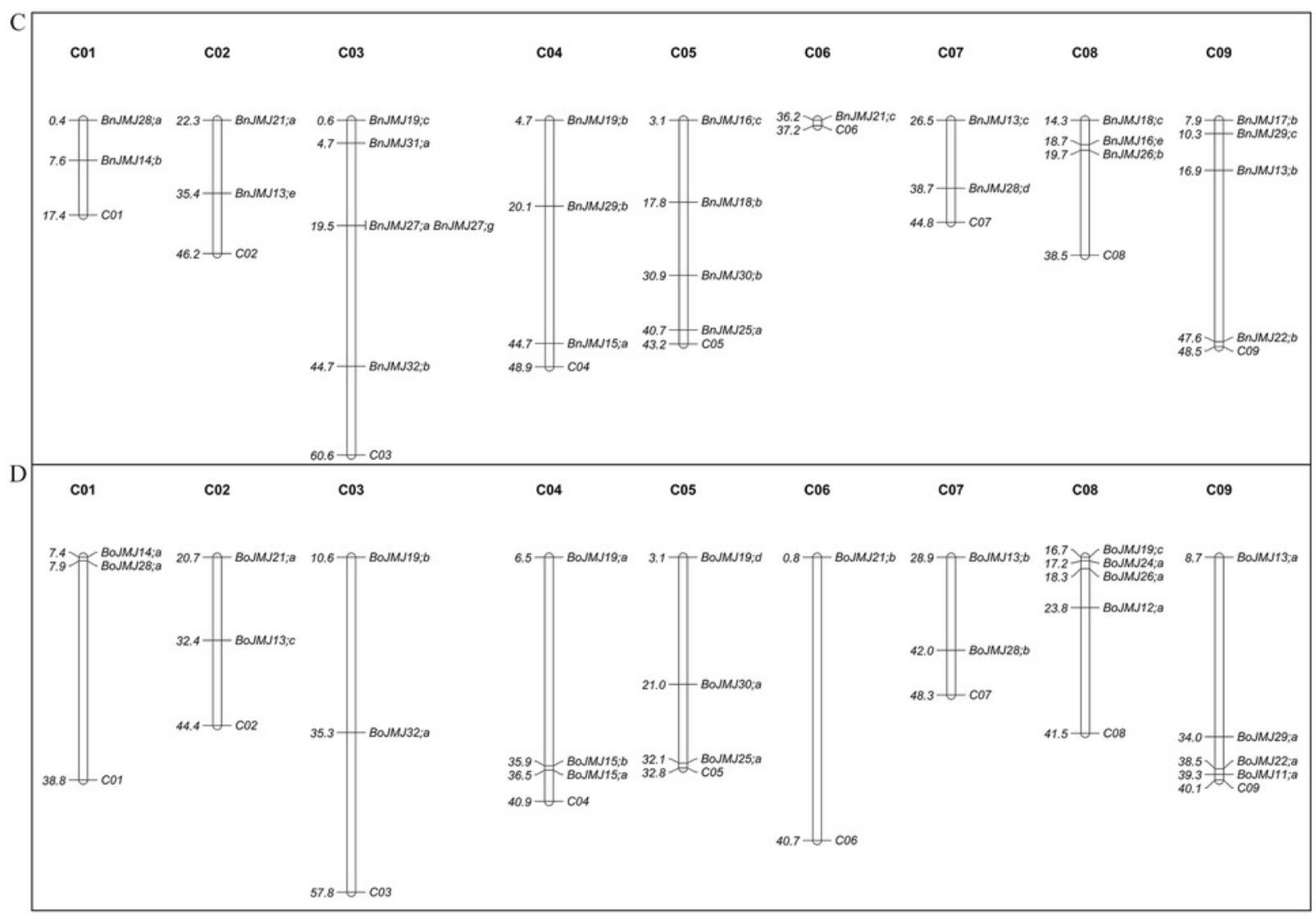




\section{Figure 2}

Fig. 2 Phylogenetic tree of JmjC domain proteins

The Phylogenetic tree included $21 \mathrm{JmjC}$ domain-containing proteins form Arabidopsis thaliana, 19 from Oryza sativa, 29 from Brassica rapa, 23 from Brassica oleracea and 65 from Brassica napus. The JmjC domain proteins can be grouped into7 groups based on the phylogenetic tree and domain organization. Different colors show different groups. JmjC domain protein sequences were aligned using ClustalW, and the phylogenetic tree analysis was performed using MEGA7.0.The trees were constructed with the following settings: tree inference as neighbor-joining; include sites as pairwise deletion option for total sequences analysis; substitution model as $p$-distance. 


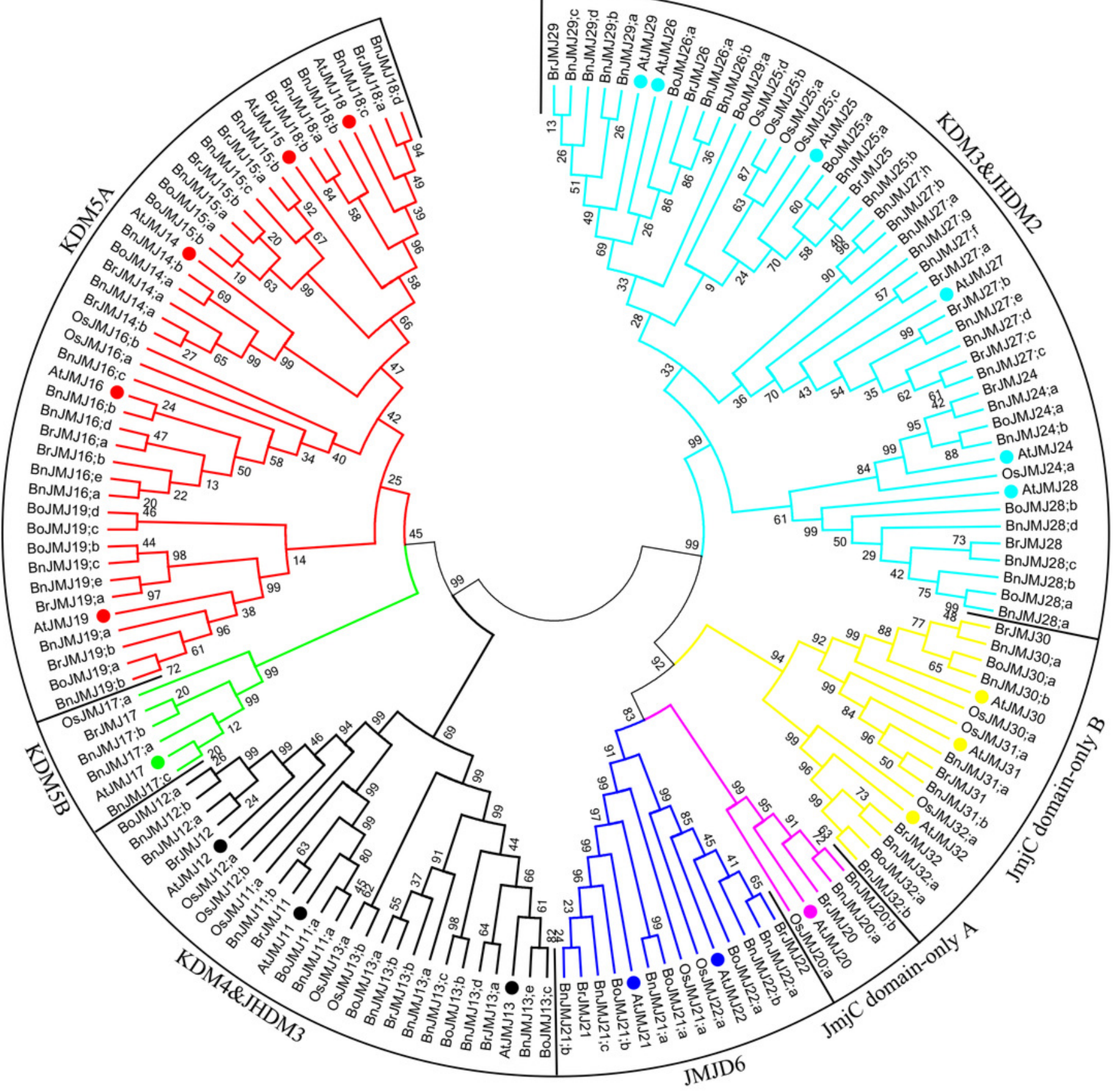


Figure 3

Fig. 3 The schematic diagrams of Group-KDM4/JHDM3
A. Phylogeny tree, B.
B. domain organization,
C. Gene structure,
D. Logos analysis of JmjC domain.
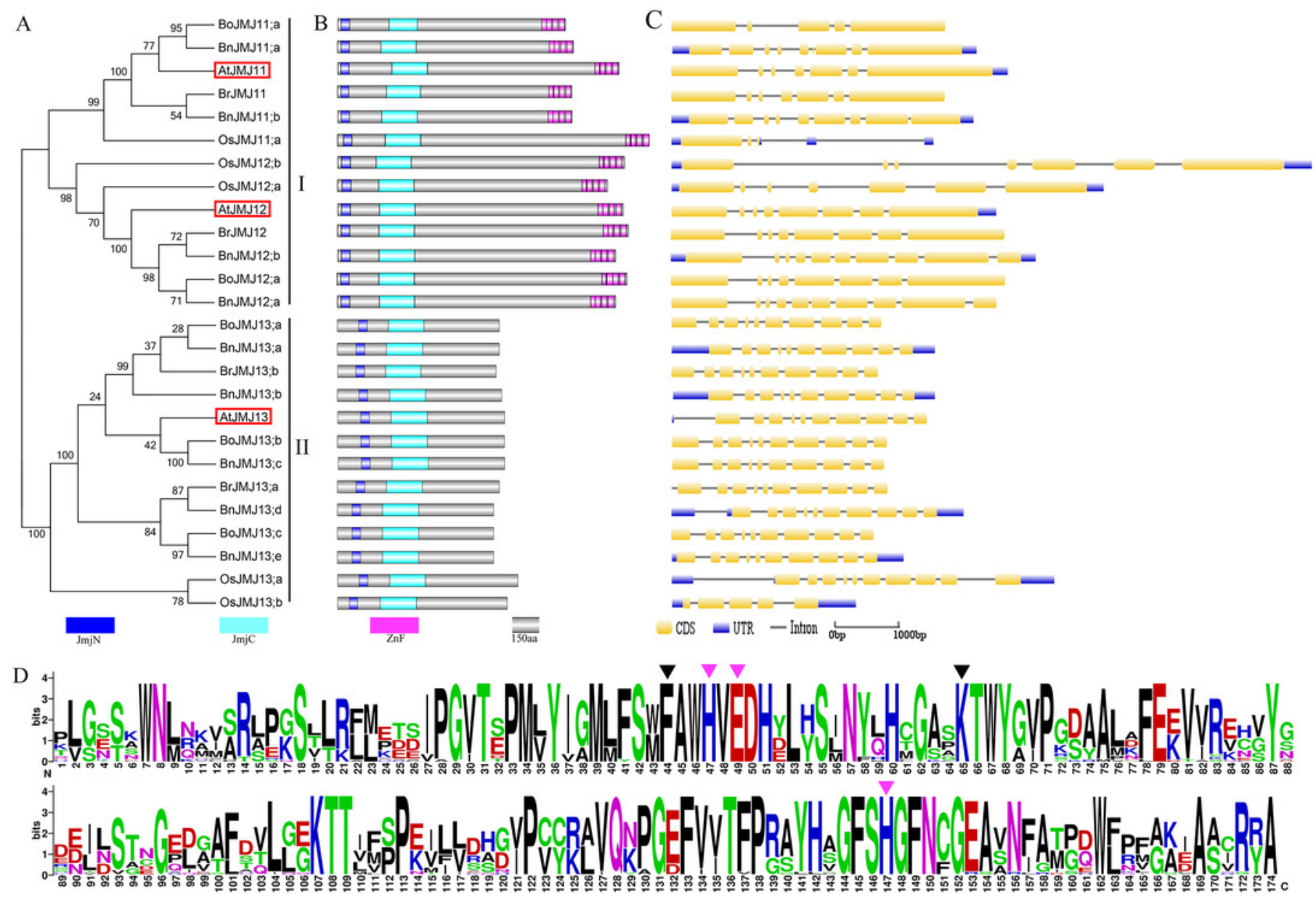
Figure 4

Fig. 4 The schematic diagrams of Group-KDM5A/B

A/E. Phylogeny tree, B/F. domain organization, C/G. Gene structure, D/H. Logos analysis of JmjC domain.
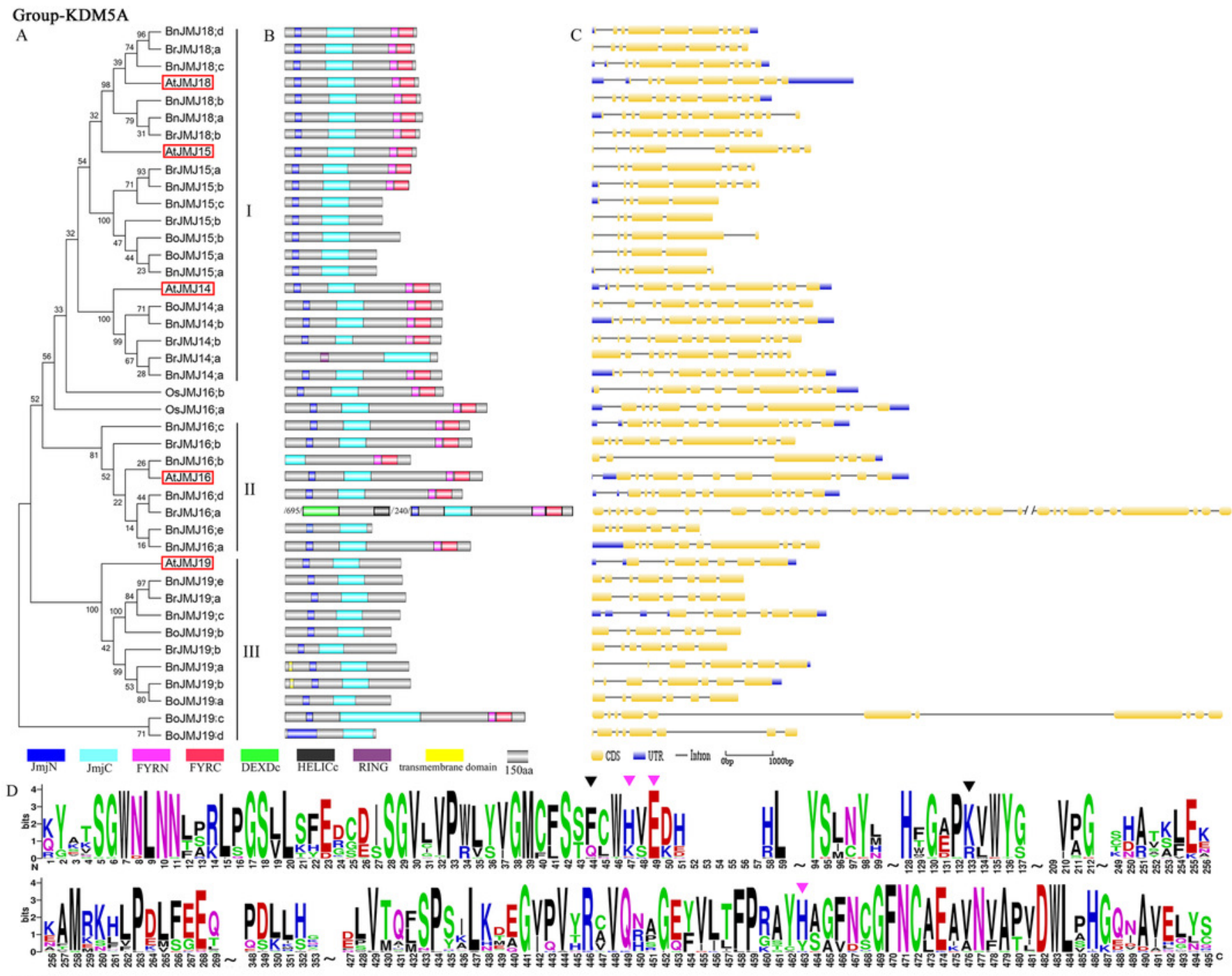

Group-KDM5B

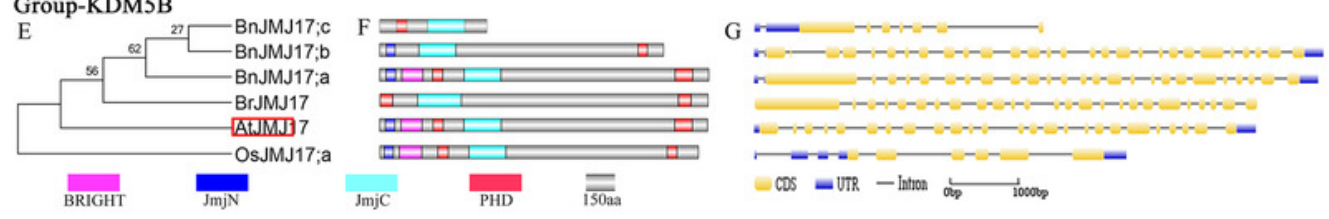

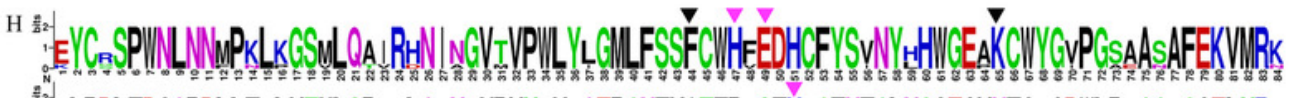

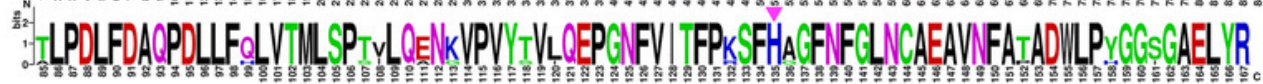


Figure 5

Fig. 5 The schematic diagrams of Group-JmjC domain-onlyA/B

A/E. Phylogeny tree, B/F. domain organization, C/G. Gene structure, D/H. Logos analysis of JmjC domain.

Group-JmjC domain-only A

A

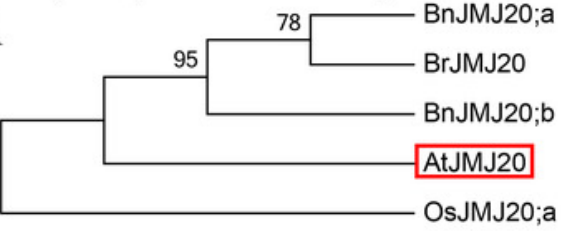

D ${\frac{\underline{z}^{2}}{\bar{a}_{1}}}_{1}$

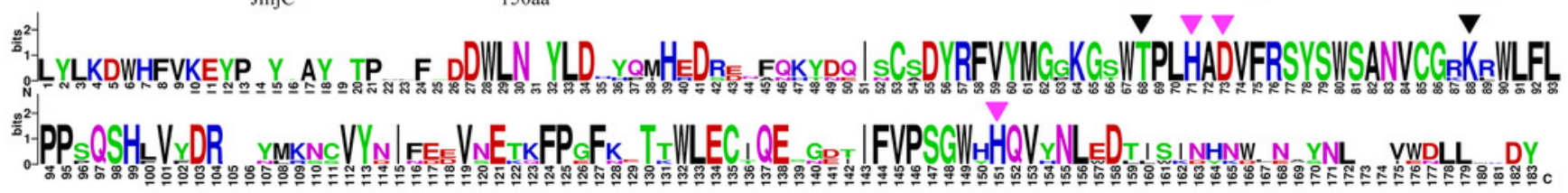

B

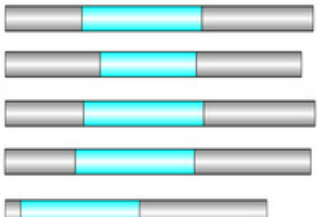

C

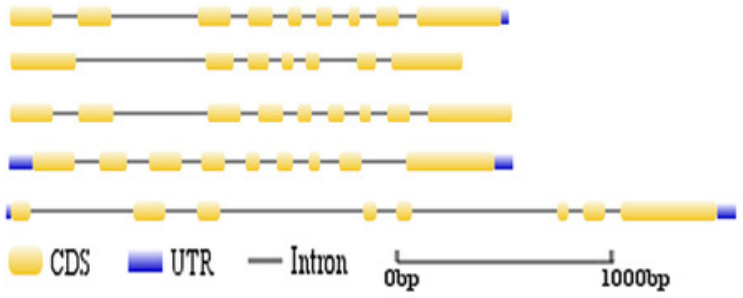

\section{$\overline{150 \text { aa }}$}

Group-JmjC domain-only B

E

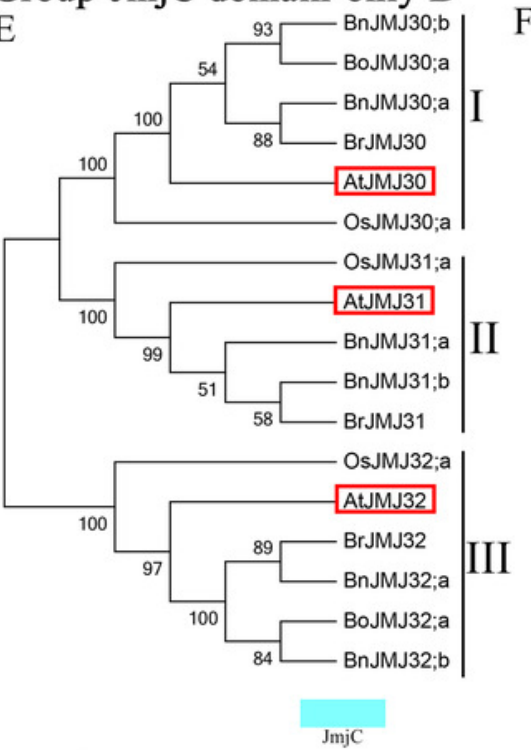

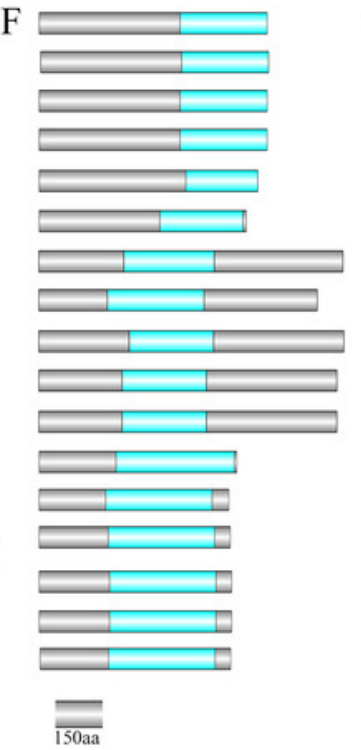

$\mathrm{G}=\ldots$

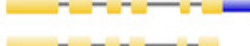

-
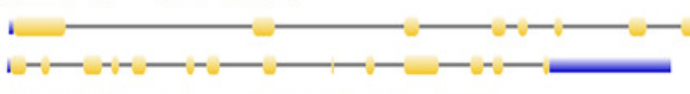

(n-1)

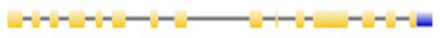

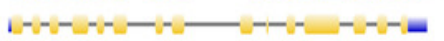

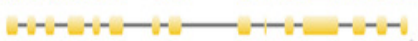

-

는

-

-

- n-

$-1--$

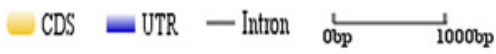

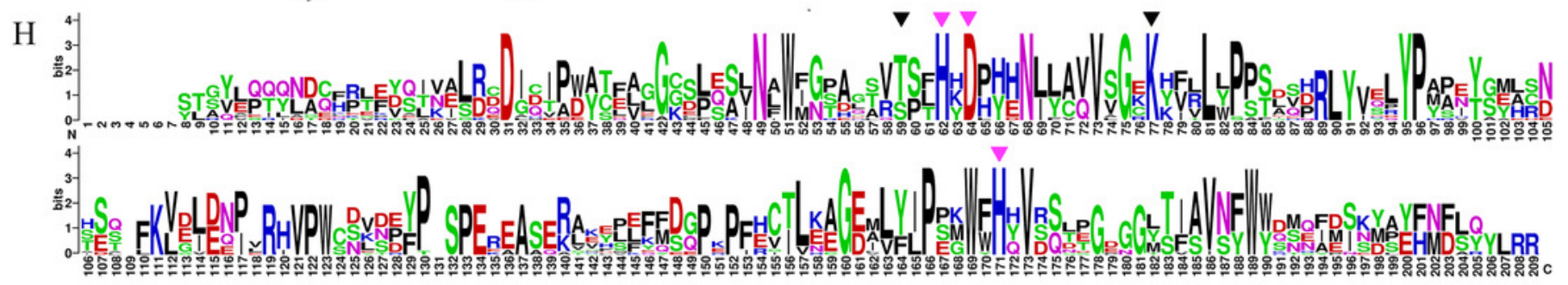


Figure 6

Fig. 6 The schematic diagrams of Group-JMJD6

A. Phylogeny tree, B. domain organization, C. Gene structure, D. Logos analysis of JmjC domain.

A

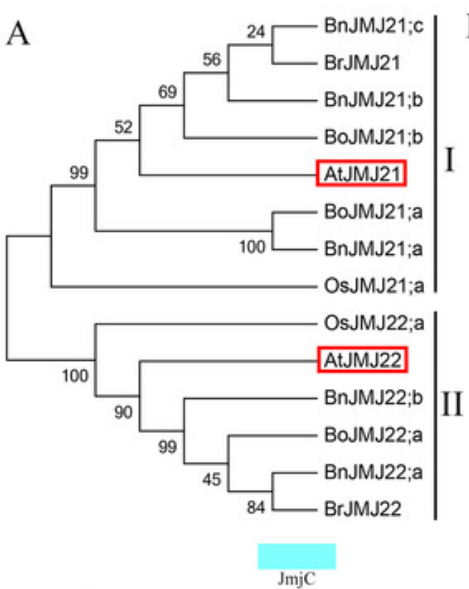

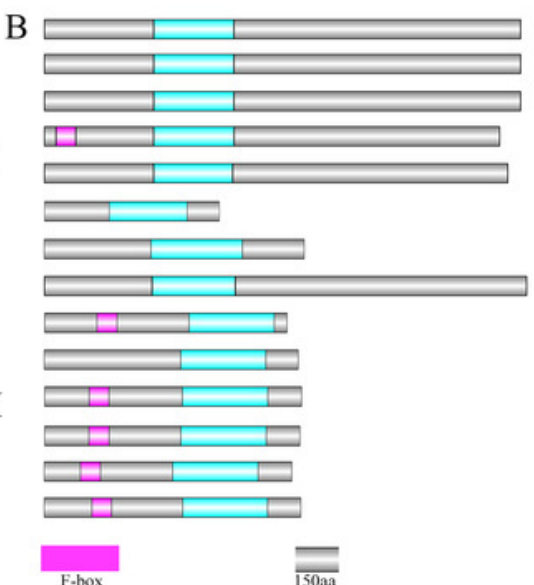

$\mathrm{C}$

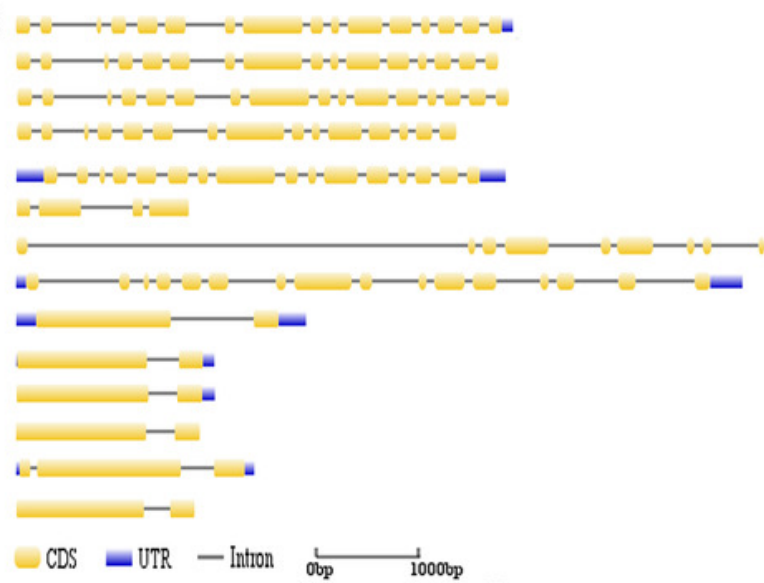

D

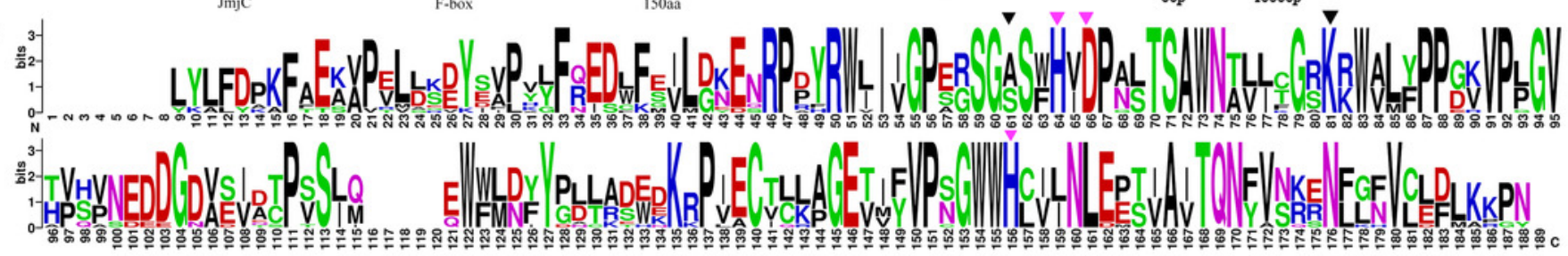


Figure 7

Fig. 7 The schematic diagrams of Group- KDM3\&JHDM2

A. Phylogeny tree, B. domain organization, C. Gene structure, D. Logos analysis of JmjC domain. 


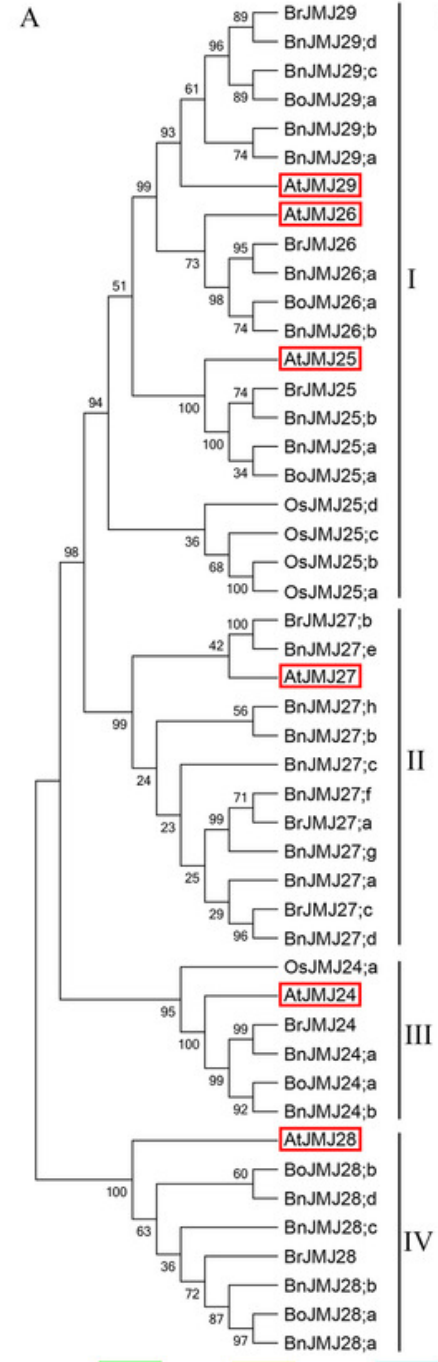

DM $\quad$ AT

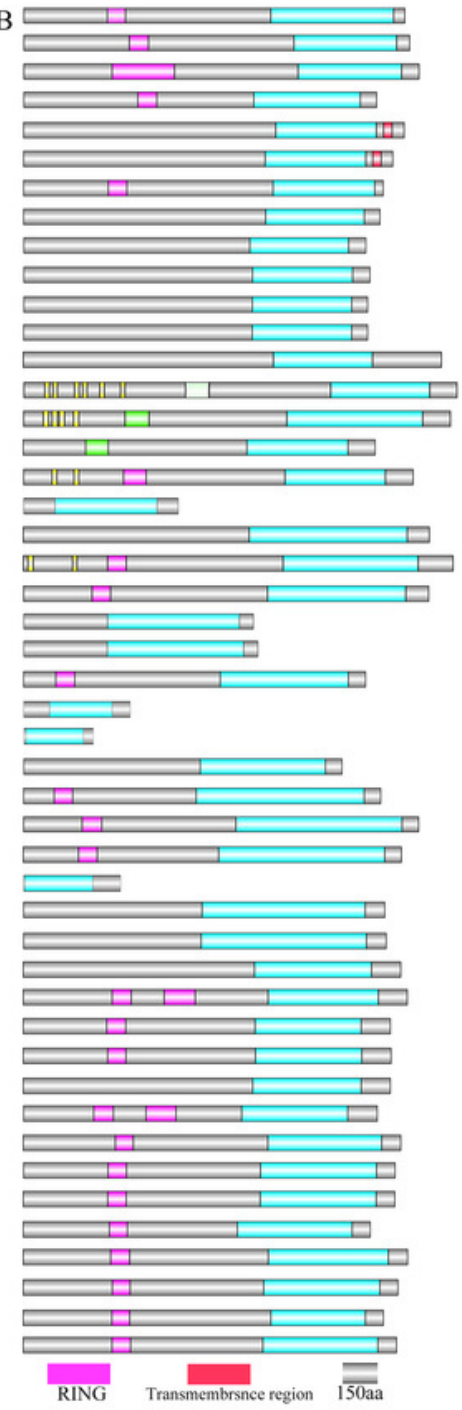

C

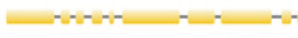

n-n $-\cdots$

0

and -

and -

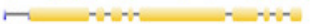

-

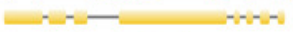

$\div-1-1-1-1$

-1- -

(1-

(n)

-

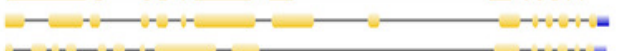

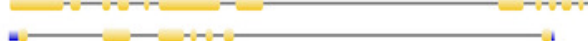

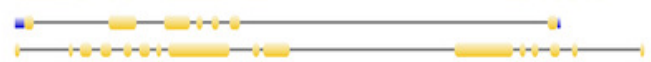

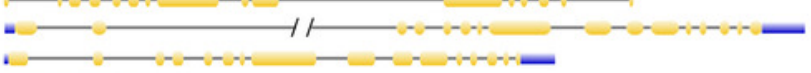

(n-

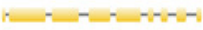

an-

- n-m-

in-

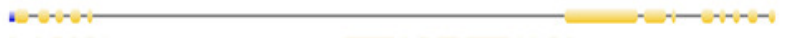

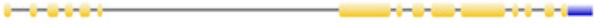

-

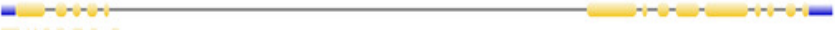

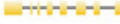

$-$

(1)-

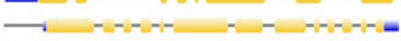

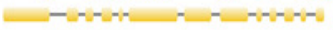

-

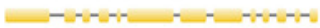

(2) -

-1- - - - - - - - -

- - - - - - n

(1) -

-

(1-

-

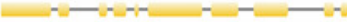

=

$\triangle$ CDS $=$ UTR - Intion oop looobp

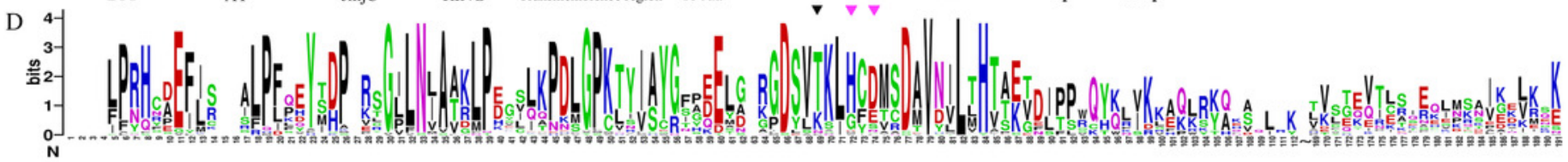

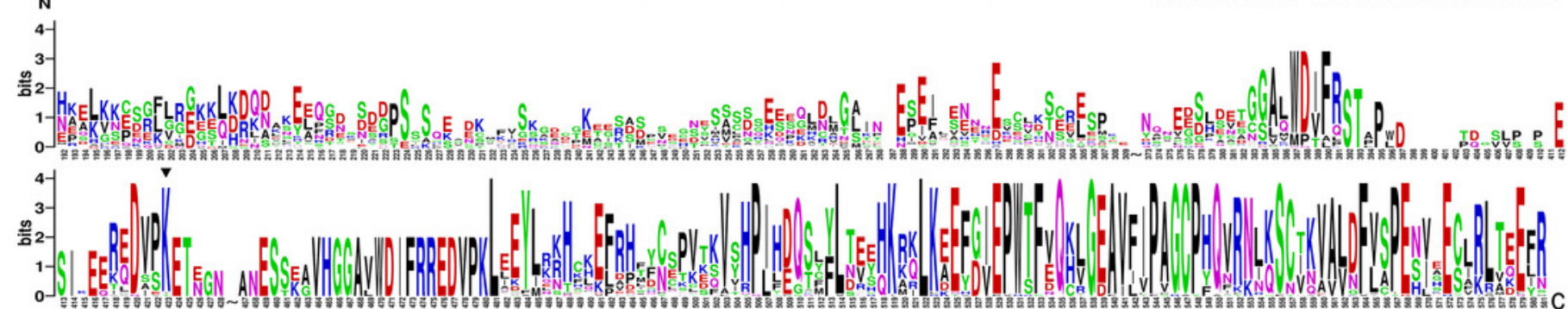


Figure 8

Fig. 8 Expression of $B$. naups KDM5 subfamily in response to drought

Many of BnJMJ14-19 genes involved in drought stress response. The error bars depict SD, an asterisk represent corresponding gene significantly up- or down-regulated by Student's t test between the treatment and the control $(0.01<P<0.05)$, two represent $(p<0.01)$
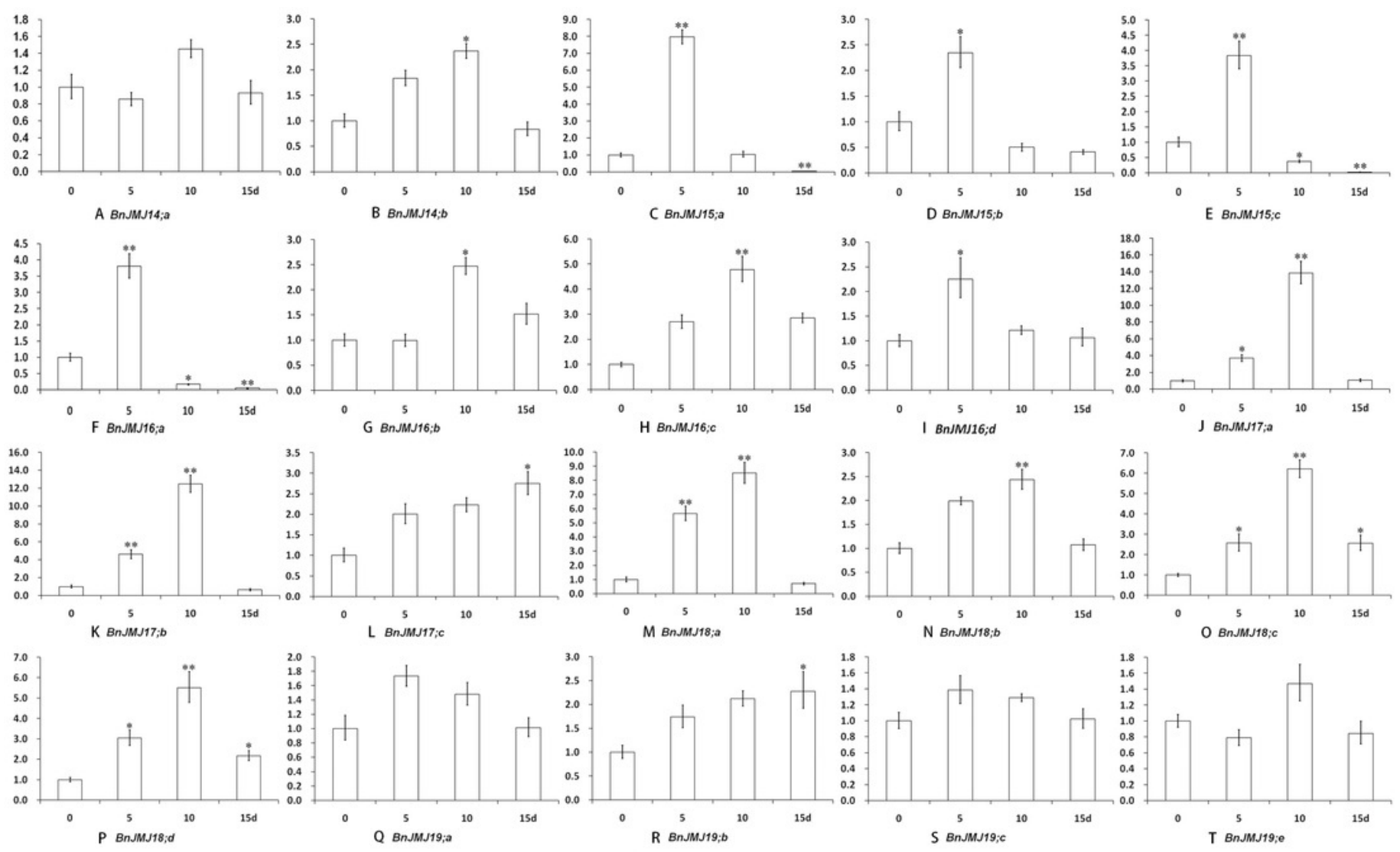

Drought stress 
Figure 9

Fig. 9 Expression of $B$. naups KDM5 subfamily in response to high temperature

Many of BnJMJ14-19 genes involved in high temperature stress response. The error bars depict SD, an asterisk represent corresponding gene significantly up- or down-regulated by Student's $\mathrm{t}$ test between the treatment and the control $(0.01<\mathrm{P}<0.05)$, two represent $(p<0.01)$
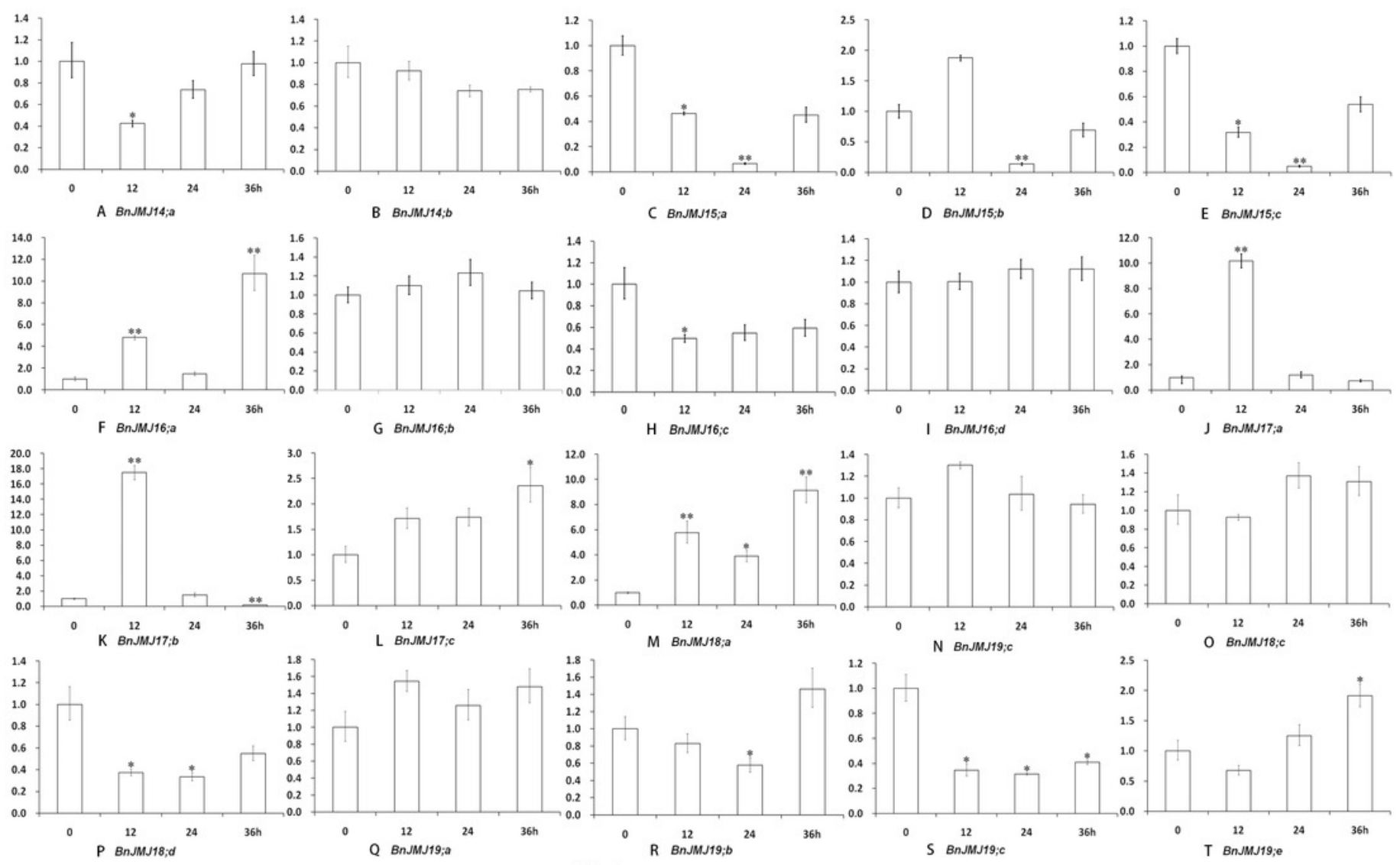

High temperature stress

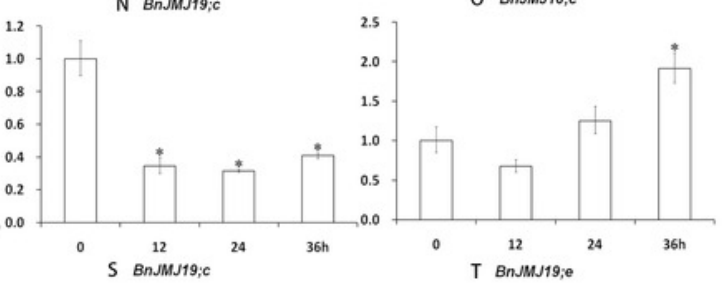


Figure 10

Fig. 10 Expression of $B$. naups KDM5 subfamily in response to $\mathrm{NaCl}$ stresses

Many of BnJMJ14-19 genes involved in NaCl stress response. The error bars depict SD, an asterisk represent corresponding gene significantly up- or down-regulated by Student's $t$ test between the treatment and the control $(0.01<P<0.05)$, two represent $(p<0.01)$
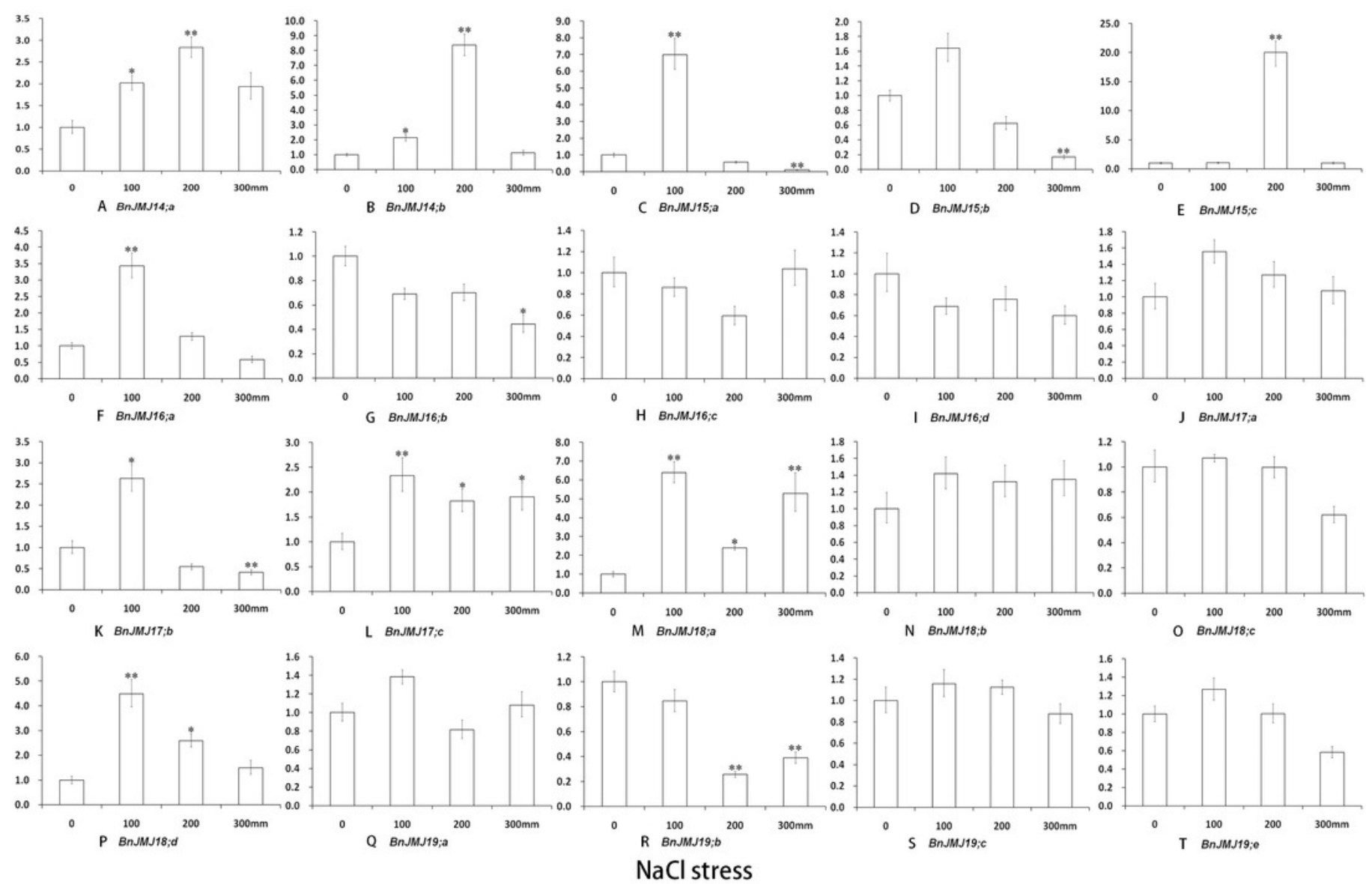